\title{
Cell wall synthesis and remodeling dynamics determine bacterial division site architecture and cell shape
}

\section{Authors}

Paula P. Navarro ${ }^{1,2, \dagger}$, Andrea Vettiger ${ }^{3, \dagger}$, Virly Y. Ananda ${ }^{1}$, Paula Montero Llopis ${ }^{4}$, Christoph Allolio ${ }^{5}$, Thomas G. Bernhardt*,3,6 , and Luke H. Chao*,1,2

\section{Affiliations}

${ }^{1}$ Department of Molecular Biology, Massachusetts General Hospital, Boston, USA

2 Department of Genetics, Blavatnik Institute, Harvard Medical School, Boston, USA

${ }^{3}$ Department of Microbiology, Blavatnik Institute, Harvard Medical School, Boston, USA

${ }^{4}$ MicRoN Core, Harvard Medical School, Boston, USA

${ }^{5}$ Faculty of Mathematics and Physics, Mathematical Institute, Charles University, Prague, Czech Republic

${ }^{6}$ Howard Hughes Medical Institute, Harvard Medical School, Boston

$\dagger$ These authors contributed equally, and sequence was determined alphabetically.

* To whom correspondence should be addressed (order determined alphabetically)

Thomas G. Bernhardt

Department of Microbiology, Harvard Medical School, Boston, USA

e-mail: thomas_bernhardt@hms.harvard.edu

Luke H. Chao

Department of Molecular Biology, Massachusetts General Hospital, Boston, USA

Department of Genetics, Harvard Medical School, Boston, USA

e-mail: chao@molbio.mgh.harvard.edu

\section{Keywords}

Bacterial cell division, Cell wall processing, Polar morphology, Cryo-electron tomography, Cryo-Focus Ion Beam milling, Fluorescence live-cell microscopy, Structured illumination microscopy 


\begin{abstract}
The bacterial division apparatus builds daughter cell poles by catalyzing the synthesis and remodeling of the septal peptidoglycan (sPG) cell wall. Understanding of this essential process has been limited by the lack of native three-dimensional visualization of developing septa. Here, we used state-of-the-art cryogenic electron tomography (cryo-ET) and fluorescence microscopy to understand the division site architecture and SPG biogenesis dynamics of the Gram-negative bacterium Escherichia coli. Our results with mutant cells altered in the regulation of SPG biogenesis revealed a striking and unexpected similarity between the architecture of $E$. coli septa with those from Gram-positive bacteria, suggesting a conserved morphogenic mechanism. Furthermore, we found that the cell elongation and division machineries are in competition and that their relative activities determine the shape of cell constrictions and the poles they form. Overall, our results highlight how the activity of the division system can be modulated to generate the diverse array of morphologies observed in the bacterial domain.
\end{abstract}

\title{
Highlights
}

- The division site architecture of $E$. coli can be modulated to resemble that of diverse bacteria.

- Cell wall degradation at the division site activates septal cell wall synthesis.

- Assembly of the cytoskeletal ring at the division site is modulated by cell wall remodeling.

- Balance between the activities of the elongation and division systems modulates cell shape. 


\section{Introduction}

3 Bacterial cells are typically surrounded by a multi-layered cell envelope that varies in complexity depending on the species ${ }^{1}$. Organisms classified as Gram-positive (monoderm) possess a single membrane layer to which a second membrane called the outer membrane (OM) is attached. Their cell wall is therefore contained in an aqueous compartment called the periplasm that is sandwiched by the inner (cytoplasmic) membrane (IM) and the $\mathrm{OM}^{2}$. Understanding the mechanisms required for the biogenesis of bacterial envelopes during cell growth and division has been a long-standing and fundamental goal of microbiology. The process is also the target of many of our most widely used antibiotics. As such, studies of cell surface assembly are of great practical significance due to the enabling insights they provide for the development of new antibacterial treatments needed to combat the growing problem of drug-resistant infections.

The cell wall is the layer of the envelope that determines cell shape ${ }^{3}$. It is also critical for fortifying the cytoplasmic membrane against osmotic lysis. The wall is assembled from the heteropolymer peptidoglycan (PG), which consists of glycan chains with repeating disaccharide units of $\mathrm{N}$-acetylglucosamine (GlcNAc) and N-acetylmuramic acid (MurNAc). Short peptides are attached to each MurNAc sugar and are used to form amide crosslinks between adjacent glycans, generating a covalently closed mesh around the cytoplasmic membrane. Because the PG layer completely envelops the cell, its expansion and remodeling are tightly coordinated with the cell cycle ${ }^{3}$.

Newborn rod-shaped cells like Escherichia coli first lengthen their cell body through the action of the elongation machinery (Rod complex, elongasome), which synthesizes and incorporates new PG material at dispersed locations throughout the cylinder ${ }^{3}$. Cell division is then initiated by the coalescence of treadmilling polymers of the tubulin-like FtsZ protein into a structure called the Z-ring at midcell ${ }^{4,5}$. The Zring is peripherally associated with the IM and its formation ultimately results in the recruitment of dozens of proteins to the prospective division site to assemble the envelope-spanning division machinery called the divisome $e^{5,6}$. A major function of this apparatus is to promote the localized synthesis of PG to generate the cross-wall/septum that divides the daughter cell compartments ${ }^{3}$. The septal PG (sPG) produced by the divisome initially connects the daughters such that it must be carefully processed to separate the newly formed cells and complete the division process ${ }^{3}$.

Our understanding of envelope biogenesis during cell division has been greatly influenced by electron micrographs of developing septa ${ }^{7}$. One of the most striking observations from these images is the extent to which the septal architectures differ between organisms ${ }^{8}$. Some of the best images of septa from a 
of Staphylococcus aureus $s^{9,10}$. In the septa of these cells, two tracks of PG material appear to be connected to the lateral cell wall, forming an electron dense $\pi$ structure with the two tracks located between the invaginating membrane. Other Gram-positive bacteria like Bacillus subtilis are only observed to produce a single, thick layer of cell wall within their septa ${ }^{11-14}$. In contrast to the flat unconstricted side wall present at Gram-positive division sites, conventional electron microscopy (EM) of fixed and stained cells and wholecell cryo-electron tomography (cryo-ET) imaging of E. coli and Caulobacter crescentus cells have visualized a V-shaped constriction in which all three envelope layers are invaginating together ${ }^{15-17 .}$ Although the general presence of SPG can be observed in some images, the existence of sample preparation artifacts and the low signal-to-noise ratio in conventional EM and cryo-ET images, respectively, have prevented a clear visualization of its structural arrangement. However, notable morphological differences between the division sites of $E$. coli and C. crescentus were evident in these images. Division sites from $C$. crescentus displayed very tight coordination between the invagination of the three envelope layers in all cells that were imaged ${ }^{16}$. They were never observed to form what would be considered a classical crosswall septum in which a band of sPG bisects the cytoplasm. By contrast, some images of $E$. coli showed a coordinately constricted envelope whereas others showed a partial crosswall where the IM and $P G$ layers seem more invaginated than the $O M^{15}$. Whether these different septal architectures reflect sample preparation artifacts, fundamental differences in the division mechanism between bacteria or arise from changes in the spatiotemporal regulation of conserved processes remains a major outstanding question.

To investigate the underlying mechanisms responsible for generating different division site morphologies, we imaged the in situ ultrastructure of the division site and the dynamics of cell envelope constriction in wild-type E. coli and several mutant derivatives with altered divisome function using cryo-ET and fluorescence microscopy. For the cryo-ET analysis, we needed to overcome the major sample thickness limitation of this imaging method in which samples $>300 \mathrm{~nm}$ in depth preclude the generation of distinguishable contrast features by $\mathrm{EM}^{18,19}$. This thickness limit is incompatible with the width of $E$. coli and most other well-studied bacterial species that have cell diameters near $1 \mu \mathrm{m}^{20}$. The development of cryo-focused ion beam (cryo-FIB) milling ${ }^{21-27}$ made this imaging possible by allowing us to generate thin, artifact-free lamellae of frozen-hydrated $E$. coli cells in various stages of division that are within the optimal thickness regime for cryo-ET.

In this work, we used cryo-FIB milling and cryo-ET for the three-dimensional (3D) visualization of E. coli septa and the SPG layer with unprecedented detail, revealing several new ultrastructural features. Micrographs of wild-type cells showed that constriction of the IM and OM was well coordinated at the start of division, but that the distance between the membranes grew in cells at later stages of division due to 
that this change in morphology arises from a more rapid constriction of the IM relative to the OM. Importantly, mutants impaired for SPG synthesis maintained coordination between IM and OM invagination throughout the division process and had division site architectures that resembled the shallowly constricted ultrastructure of $C$. crescentus. Moreover, mutants defective for SPG remodeling were shown to be largely blocked for OM constriction and their septa as well as those of wild-type cells were found to contain lateral bridge structures and separate parallel plates of SPG reminiscent of septa from S. aureus. In addition to tracking IM and OM dynamics, we also monitored the rates of SPG synthesis and remodeling at the division sites of the various mutants and correlated them with the rates of lateral wall biogenesis, division site ultrastructure, and the shape of daughter cell poles. Our results revealed that cell wall degradation at the division site plays a role in both the activation of SPG synthesis and Z-ring condensation, suggesting communication between the PG synthesis machinery on one side of the membrane and the cytoskeleton on the other. Furthermore, this analysis provided compelling evidence that the cell elongation and division machineries are in competition for shared PG precursors and that the relative activities of the two systems determines the shape of division site constrictions and the poles they form. Overall, our results highlight the plasticity of the division system and how modulation of its cell wall synthesis and remodeling activities can help generate the array of cell and polar morphologies observed in the bacterial domain. 
Results

In situ architecture of the $E$. coli cell envelope at the division site

Most of the available images of the division site architecture in E. coli come from conventional EM micrographs of fixed and stained cells ${ }^{28}$. Although these images have given us a general idea of how the cell envelope is remodeled during division to build the daughter cell poles, the division site ultrastructure observed depended greatly on the fixation conditions used. Some treatments yielded division sites with a uniformly constricted architecture, whereas others captured cells with a partial crosswall septum composed of a fold of IM sandwiching a stretch of SPG from which the OM was excluded ${ }^{15,29}$. In contrast, whole-cell cryo-ET images of E. coli grown in nutrient poor conditions to reduce their size have thus far only shown uniformly constricted septa without a clear view of the SPG layer ${ }^{17}$. The variation in septal architecture based on the imaging method or fixation condition used has made it difficult to draw definitive conclusions about which architecture represents the native state of the division site. We therefore reinvestigated the ultrastructure of the E. coli division site using cryo-FIB milling to obtain thin ( $150-200 \mathrm{~nm}$ thick) lamellae of dividing cells for subsequent in situ cryo-ET imaging (Fig. S1). A total of 9 tilt-series of wild-type cells were acquired, aligned, and 3D reconstructed (Table S1). Additionally, to gain better visualization of SPG, non-linear anisotropic diffusion (NAD) filtering was applied to denoise the cryo-electron tomograms. Using the measured distance between the leading edge of the IM constriction for each image, they were grouped into three classes representing what appeared to be different stages of division: (i) constriction, (ii) septation, or (iii) cytokinesis (Fig. 1A). Cells with an IM-IM distance of around $500 \mathrm{~nm}$ were classified as being in the constriction phase. They had a V-shaped constriction with a relatively uniform invagination of the two membranes and an indented mesh of PG (Fig. 1A-B, and S2A). The second category of cells had a much smaller IM-IM distance $(50 \mathrm{~nm})$. They displayed a partial septum in which the IM was observed to be more deeply invaginated than the OM with an average difference of $134.1( \pm 21) \mathrm{nm}$ (mean \pm SD) in their measured OM-OM versus IM-IM distance (Fig. 1A-B, and S2B). Strikingly, the denoised tomograms showed an elongated, triangular wedge of PG filling the gap between the two membranes (Fig. 1B). This structure is reminiscent of the lateral bridge in $S$. aureus septa connecting the plates of $\mathrm{sPG}^{10}$, except in this case a network of electron densities is visible connecting the two plates of PG that will become the daughter cell polar PG caps. In cells at the final stage of cytokinesis, IM fission was observed to be completed, leaving cells connected by the two interwoven SPG plates. The OM of these cells displayed deep constrictions with the leading edge of the membrane pointing between the two sPG plates, presumably poised to traverse the remaining OM-OM distance of 100-250 nm to complete division (Fig. 1A-B, S2C and Video S1). No OM blebs or cytoplasmic mesosome-like structures were observed at the division site in the cryo-ET data like those observed previously by conventional EM ${ }^{15,28}$. 
124 The different division site architectures observed in the cryo-ET analysis suggested that the constriction rate of the IM exceeds that of the OM during division to promote the formation of the partial septa. To investigate this possibility, we followed the constriction dynamics of each membrane using live-cell fluorescence microscopy. The IM was tracked using a superfolder GFP (sfGFP) fusion to the IM-anchored division protein ZipA (ZipA-sfGFP) whereas constriction of the OM was followed using mCherry fused to the OM-localized lipoprotein Pal (Pal-mCherry) (Fig. 1C and Video S2). Analyzing the distribution of the fluorescence signals of Pal-mCherry and ZipA-sfGFP at the division site confirmed the further constriction of IM with respect to the OM (Fig. 1D) as seen by cryo-ET (Fig. 1A-B). Following time-lapse imaging of a

132 large population of cells $(N=150)$, the invagination rate of each membrane was calculated from 133 kymographs (Fig. 1E-F). We found that the IM constricted at a rate of $64.26 \pm 33.98 \mathrm{~nm} / \mathrm{min}$ and the OM 134 at a rate of $55.03 \pm 23.25 \mathrm{~nm} / \mathrm{min}$. These results are in-line with previously measurements of cytokinesis 135 rates $^{30,31}$. Multiplying the difference in constriction velocity between the membranes by the average time 136 for cytokinesis (16.36 $\pm 7.49 \mathrm{~min}$ ) predicts that the IM-IM versus OM-OM diameters will differ by upwards 137 of $147 \mathrm{~nm}$ at late stages in cell division, which is in good agreement with our cryo-ET data (Fig. S2). Thus, 138 the combined cryo-ET and time-lapse analysis definitively establish that $E$. coli divides by a mixed 139 constriction/septation mechanism. At early stages in the process, invagination of the three envelope layers 140 is well-coordinated, resulting in a V-shaped constriction architecture. However, because the IM invaginates 141 about $9 \mathrm{~nm} / \mathrm{min}$ more rapidly than the OM, the distance between the two membranes grows as division 142 progresses and a partial septum with a structure of sPG reminiscent of some Gram-positive bacteria is 143 formed. Thus, the division site architectures of diverse bacteria appear to share more similarities than 144 previously anticipated.

\section{sPG synthesis and remodeling activities of the divisome define division site architecture}

We next wanted to determine how the architecture of the division site and the dynamics of its constriction are altered by mutations affecting SPG synthesis and remodeling. The essential PG synthase of the divisome is formed by a complex between FtsW and Ftsl (FtsWI) (Taguchi et al., 2019). Following Z-ring assembly at the prospective division site, a regulatory pathway is thought to be initiated that ultimately results in the activation of SPG synthesis by this synthase ${ }^{34-37}$ (Fig. 2A). Activation is mediated in part via a direct interaction between FtsWI and the FtsQ-FtsL-FtsB (FtsQLB) complex ${ }^{38}$. Although the mechanism is unclear, genetic evidence suggests that the FtsQLB activation event is stimulated by an essential peptide within the division protein $\mathrm{FtsN}^{39}$. Another domain of $\mathrm{FtsN}$ called the SPOR domain is responsible for concentrating the activation peptide at the division site through its ability to bind SPG that has been processed by PG cleaving enzymes called amidases ${ }^{39-41}$. The amidases remove the stem peptides from the glycan strands as they split the SPG septum to promote OM constriction and daughter cell separation ${ }^{42}$. In the process, they generate the denuded PG glycan strands recognized by the SPOR domain ${ }^{40,41}$. The 
160

161

162

163

164

165

166

167

168

169

170

171

172

173

174

175

176

177

178

179

180

181

182

183

184

185

186

187

188

189

190

191

192

193

194

195

recruitment of more Fts $\mathrm{N}$ to the division site has been proposed to generate a positive feedback loop, the sPG loop, that drives the activation of SPG biogenesis and cell division ${ }^{39}$. To modulate this process for our analysis, we employed several different mutant strains (Table S2-S3): (i) a mutant lacking the SPOR domain of FtsN (ftsN- $\triangle S P O R)$, (ii) mutants defective for one ( $\triangle e n v C)$ or both ( $\triangle e n v C \Delta n l p D)$ activators required for amidase activity ${ }^{43}$, and (iii) a mutant ( $f t s L^{*}$ ) encoding a variant of FtsL that hyperactivates $S P G$ synthesis by $\mathrm{FtsWI}$ and bypasses the requirement for $\mathrm{FtsN}^{37}$ (Fig. 2A). A comprehensive gallery of cryoelectron tomograms containing the analyzed E. coli strains is provided (Fig. S3). A total of 60 tomograms were obtained and analyzed in this study (Table S1).

Division sites from cells deleted for the SPOR domain of FtsN resembled those observed previously for $C$. crescentus $^{16}$ (Fig. 2B). By cryo-ET, there appeared to be greater coordination between IM and OM constriction throughout the division process with the IM-OM spacing in deeply constricted cells $(79.2 \pm 21.7$ $\mathrm{nm}$ ) measuring nearly half that of wild-type cells in the septation phase (Fig. 2B, S2-3, Video S3). Fluorescence microscopy tracking of $\mathrm{IM}$ and $\mathrm{OM}$ constriction dynamics confirmed that the rate of constriction for the two membranes was nearly identical in the fts $N-\triangle S P O R$ mutant (Fig. 2C-D, Video S2). In this case, the rate of IM constriction was reduced compared to wild-type cells (49.56 \pm 13.93 versus $64.26 \pm 33.98 \mathrm{~nm} / \mathrm{min}$ ) such that it more closely matched the rate of OM constriction (Fig 2D). As a result of the close opposition of the IM and OM, the wedge of SPG observed in filtered tomograms was not as elongated as in wild-type cells such that separate plates of material forming ahead of the wedge could not be clearly visualized (Fig. 2B).

In contrast to the coordinated envelope constriction displayed by the $f t s N-\triangle S P O R$ mutant, cells defective for both amidase activators $(\Delta e n v C \Delta n l p D)$ formed a Gram-positive-like septum in which the constriction of the IM was completed without much observable invagination of the OM (IM-OM distance: $296.05 \pm$ $133.45 \mathrm{~nm}$ in deeply constricted cells) (Fig. 2B and S3). A similar septal architecture was observed previously for this mutant using conventional EM, but the ultrastructure of the SPG layer was not preserved $^{44}$. NAD-filtering of the current cryo-electron tomograms revealed density corresponding to SPG that was even more clearly discernable as two distinct plates of material than in the partial septa observed in wild-type cells above (Fig. 1B and 2B). Furthermore, the tomograms also revealed a triangular wedge of $P G$ material at the outer edges of the septa that was not previously observed in the conventional EM analysis and presumably serves as a roadblock to OM invagination (Fig. 2B, Video S4). It was not possible to measure membrane constriction dynamics in live cells of the $\Delta e n v C \Delta n l p D$ double mutant due to its poor growth. However, measurements in a mutant lacking only EnvC, the most important of the two amidase activators $^{43}$, revealed a significant disparity between IM and OM constriction rates (Fig. 2C-D. Video S2). Even though the IM constriction rate was slower than wild-type or $\mathrm{ftsN}-\Delta S P O R$ cells $(33.97 \pm 18.42 \mathrm{~nm} / \mathrm{min})$ the completion of IM invagination still preceded that of the OM by an average of $36.5 \mathrm{~min}$ (Fig. S4). Overall, 
196

197

198

199

200

201

202

203

204

205

206

207

208

209

210

211

212

213

214

215

216

217

218

219

220

221

222

223

224

225

226

227

228

229

230

231

the results with the fts $\mathrm{N}-\triangle S P O R$ and amidase activation mutants indicate that impairing key components of the divisome involved in SPG synthesis activation and remodeling alters the architecture of the $E$. coli division site such that it begins to resemble that of other distantly related bacteria.

\section{Hyperactivated SPG biogenesis leads to aberrant division site architecture}

To determine the effects of hyperactivated SPG biogenesis on division site architecture, we imaged cells of the $\mathrm{fts} L^{*}$ mutant. Strikingly, cryo-ET revealed an altered architecture in which the density corresponding to the wedge of SPG observed in wild-type and other mutant cells was missing, and the envelope at the leading edge of the invagination was 48\% thinner than in wild-type (Fig. 2B and Fig. S5B). Conversely, the envelope in the nascent polar regions adjacent to the leading edge of the invagination was $15 \%$ thicker in $\mathrm{ftsL}^{*}$ cells than in wild-type with the bulged areas appearing to contain more PG than normal (Fig. S5B and Video S5). As expected from the faster division time measured previously for $\mathrm{ftsL}^{*}$ mutant $^{37}$, live-cell imaging showed that the constriction velocity of the IM was much greater than for wild-type cells (86.18 \pm $33.12 \mathrm{~nm}$ versus $64.26 \pm 33.98 \mathrm{~nm} / \mathrm{min}$ ) (Fig. 2C-D, Video S2). Surprisingly, the rate of OM constriction was measured to be much slower than that of the IM (42.60 $\pm 18.49 \mathrm{~nm}$ versus $55.30 \pm 23.25 \mathrm{~nm})$ (Fig. 2C-D), a difference that would normally be expected to give rise to cells with partial septa with large distances between the IM and OM. However, partial septa were not observed in the tomograms (Fig. 2B). The distances between the IM and OM remained relatively constant in all the cells that were imaged (Fig. 2B and Fig. S2-3). This discrepancy is likely due to the PG binding activity of Pal causing the Pal-mCherry OM reporter to get stuck in the thicker PG that accumulates behind the closing septum and therefore to track poorly with the leading edge of the invaginating OM in these cells. In contrast, cryo-ET data directly visualize OM. Notably, upon closer inspection of kymographs of the $\mathrm{fts} \mathrm{L}^{*}$ mutant, we noticed that one side of the cell constricted much faster than the other (Fig. 2C), a phenomenon that was also observed by cryoET (Fig. S6A-C). Applying Fourier filtering to kymographs allowed us to distinguish between forward and reverse signal trajectories in order to compare the constriction velocity for each side of the division site and assess the degree of anisotropy during septum closure. We found that $f t s{ }^{*}$ showed a higher but not statistically significant (Kruskal-Wallis ANOVA, $p=0.056$ ) anisotropy score for both IM and OM constriction compared to the other strains (Fig. S6B-C). Additionally, when cells were imaged in vertical orientation, the constriction of $f t s L^{*}$ cells was observed to be less circular than in wild-type as expected for uneven closure of the division ring (Fig. S6D). Thus, circumvention of the normal controls regulating SPG biogenesis in the $f t s L^{*}$ mutant results in aberrant division site geometry and abnormal thickening of the envelope at the cell poles. These cells also lack an observable sPG wedge, which may destabilize the division site and help explain why these mutants were originally found to lyse at elevated temperatures ${ }^{45,46}$. 
sPG degradation activates its synthesis

233 To better understand the mechanism(s) by which the observed changes in division site architecture are 234 caused by mutations altering divisome components, we measured the rates of sPG synthesis and degradation in $\mathrm{fts} L^{*}, \mathrm{fts} N-\triangle S P O R$, and $\triangle e n v C$ cells relative to those of wild-type using two different cytological assays (Fig. 3 and S7). The first assay used a pair of compatibly labeled fluorescent D-amino acids (FDAAs), YADA and HADA ${ }^{47}$. In E. coli, these probes are primarily incorporated into the peptide stem of PG by alternative crosslinking enzymes called L,D-transpeptidases (LDTs) ${ }^{48}$. Because they do not use the canonical PG synthases (e.g. FtsW, PBP1b, etc.) for incorporation, FDAAs only provide an indirect readout of nascent PG synthesis. Nevertheless, when two probes are used for labeling in sequence, they can accurately label areas of newly synthesized material ${ }^{49}$. In our experiments, we grew cells for an extended period in the presence of YADA to label the entire PG sacculus. A portion of these cultures was then fixed to establish the YADA labeling baseline while other portions were washed, pulsed with HADA for different lengths of time, and then fixed prior to visualization. The intensity of the HADA signal that appeared at midcell after the pulses was used as a measure of the rate of SPG insertion (Fig. 3A). Additionally, the YADA label was used to follow the fate of old PG with a comparison of the signal intensity at midcell before and after the HADA pulse providing a measure of SPG degradation (Fig. 3A). To measure nascent PG synthesis directly, the second assay employed a MurNAc-alkyne probe ${ }^{50}$ in conjunction with HADA (Fig. S7). In this case, cells were pre-labeled with HADA to mark old PG and pulsed with MurNAcalkyne, which is incorporated into the PG precursor lipid II and used directly by canonical synthases to build new $P^{30}$. Thus, using click chemistry and a clickable fluorescent dye, the rate of sPG insertion was measured from the MurNAc-alkyne labeling intensity, and comparison of the HADA signal before and after the pulse was used to determine the rate of SPG degradation.

Both PG biogenesis assays yielded qualitatively similar results (Fig. 3 and S7), and the measurements of SPG synthesis correlated well with the rates of IM invagination determined above (Fig. 2D). The ftsL* mutant synthesized SPG faster than all other strains just as it had the fastest rate of IM invagination (Fig. 2D, 3B, and S7D). This result confirms that activated FtsQLB complexes indeed hyperactivate sPG synthesis as suggested by their recently reported effects on the dynamic motions displayed by the FtsWI PG synthase ${ }^{30}$. Notably, the dual FDAA assay detected a significant amount of old PG at the division sites prior to the HADA pulse, and this material appeared to be relatively stable during the time course. Additionally, bright foci of old material were also observed at the poles of many cells after extended YADA labeling (Fig. 3B S8A-B). This accumulation of old material likely corresponds to the thickened areas of cell wall in the curved regions of septa and nascent poles observed by cryo-ET of the ftsL * mutant (Fig. S5 and S8C-D), reinforcing the conclusion that short-circuiting the normal controls governing the activation of SPG biogenesis not only leads to more rapid SPG synthesis and septal closure, but also results in the aberrant accumulation of PG within the developing cell poles. 
As suggested by their slower than normal rates of IM invagination, both the fts $\mathrm{N}-\triangle S P O R$, and $\triangle e n v C$

mutants displayed a reduced rate of SPG synthesis relative to wild-type cells (Fig. 3C and S7D). In the MurNAc-alkyne assay, the $f t s-\triangle S P O R$ mutant also showed a reduced septal labeling efficiency (Fig. S7C), which is another indication of reduced SPG synthesis activity ${ }^{30}$. Although the sPG synthesis rates were similar, the two mutants differed greatly in their rates of sPG degradation. The ftsN- $\triangle S P O R$ mutant displayed relatively normal rates of SPG degradation whereas $\triangle e n v C$ cells showed reduced turnover of SPG as expected for a mutant lacking an important amidase activator (Fig. 3D-E and S7E). The combination of slower SPG synthesis with relatively normal SPG degradation explains the well-coordinated constriction phenotype displayed by the fts $N-\triangle S P O R$ mutant in the cryo-ET analysis. Although reduced SPG degradation was expected for the amidase activation mutant based on its cell chaining phenotype and abnormal septal morphology, it has not been directly demonstrated previously. The reduced rate of SPG synthesis in the $\triangle e n v C$ cells is notable, however, because it indicates that proper sPG processing by the amidases is required for normal rates of SPG synthesis. This result along with the reduced rate of SPG synthesis observed for the ftsN- $\triangle S P O R$ mutant provides strong support for the SPG loop model for PG biogenesis during division, which so far has been based solely on inferences from a genetic analysis of the domain functions of $\mathrm{FtsN}^{39}$.

\section{sPG degradation is required for normal Z-ring formation}

286 In the FDAA labeling experiments we noticed that cells lacking EnvC commonly displayed closely spaced sPG labeling consistent with the aberrant formation of adjacent division sites (Fig. 4A). Accordingly, closer examination of the localization of Pal-mCherry in these cells revealed that double bands of the OM marker for sites of cell constriction occurred at a significantly elevated frequency over wild-type or fts $N-\triangle S P O R$ cells (Fig. 4B, E-F). These double constrictions were typically observed within the cell body, but we also observed constrictions near cell poles, generating what appeared to be minicells (Fig. 4C, G). However, free minicells were not observed in the culture, suggesting that these aberrant poles were likely generated from a double constriction event within the cell body, one of which was aborted while the other completed division to leave behind a daughter with a polar constriction. Another defect in division we noticed in the $\triangle e n v C$ cells was the formation of membrane blebs emanating from some of the developing septa (Fig. 4C). Cells with such blebs were often observed to lyse, suggesting there was a catastrophic failure in division. Lysed cells and double constrictions were also observed in the cryo-ET analysis of $\Delta e n v C \Delta n / p D$ cells (Fig. 4D).

In addition to the aberrant sPG and Pal-mCherry localization, the pattern of ZipA-sfGFP was also altered in the $\triangle$ envC mutant relative to wild-type and the other mutants (Fig. 4H, Video S6). ZipA is an FtsZbinding protein and a marker for the Z-ring ${ }^{51}$. Many ZipA-sfGFP structures in cells lacking EnvC were 
cells (Fig. 4H). Consistent with this possibility, averaging the ZipA-sfGFP signals for a population of cells $(\mathrm{N}=100)$ either over a 10 -minute time window or over a $2 \mu \mathrm{m}$ volume spanning midcell showed that the fluorescent signal was more broadly distributed in the $\Delta e n v C$ mutant than in wild-type cells or cells of the other mutants (Fig. 4l-J). Notably, this phenotype was not suppressed by combining the sPG synthesis activating $f t s L^{*}$ mutation with $\triangle e n v C$, indicating that it likely stems from the loss of sPG processing not its collateral effect on SPG synthesis (Fig. 4H-J). A similar diffusion of the Z-ring signal has been observed for cells defective for FtsZ-binding proteins like ZapA that are thought to bundle FtsZ polymers to condense the ring ${ }^{52,53}$. These results therefore suggest a previously unappreciated role for SPG hydrolysis by the amidases in the periplasm in promoting Z-ring condensation in the cytoplasm. The observation of double septa and failed division sites also indicates an unexpected role for amidase activation in division site stability and/or placement in addition to their known function in cell separation (see Discussion).

\section{Competition between elongation and SPG biogenesis determines polar shape}

The idea that the cell elongation and division machineries might be in competition with one another was proposed many years ago ${ }^{54,55}$. However, experimental support for such a competition has been limited, and it has never been demonstrated that competition occurs at the level of PG biogenesis. To investigate this possibility further, we took advantage of the PG labeling assays used to measure sPG biogenesis to also quantify the incorporation of new PG material into the sidewall (cell cylinder) during the MurNAcalkyne or HADA pulses (Fig. 3F and S7F). Importantly, we found that sidewall PG and SPG synthesis were inversely proportional in the different strains we studied. Sidewall PG incorporation was highest in the ftsN$\triangle S P O R$ mutant, which had one of the lowest rates of SPG synthesis (Fig. 3 and S7). Conversely, sidewall $P G$ synthesis was the lowest in the $f t s L^{*}$ mutant that displayed the highest measured rates of SPG production (Fig. 3 and S7). In addition to the rates of sidewall PG biogenesis being inversely proportional to that of SPG biogenesis, they were also found to be directly related to the single cell elongation rates of the division mutants, with fts $N-\triangle S P O R$ mutants elongating 1.5 times faster than $f t s L^{*}$ cells (Fig. 5A). Another measure of cell elongation activity is the circumferential motions of MreB and other components of the Rod complex around the cell cylinder ${ }^{56-58}$. We therefore tracked the motion of a mNeonGreen fusion to MreB in wild-type and mutant cells using a combination of structured Illumination microscopy and total internal reflection fluorescence (SIM-TIRF) imaging. Consistent with the sPG synthesis measurements, the total number of directionally moving MreB filaments per area was significantly reduced in $f t s L^{*}$ and $\triangle$ envC cells (Fig. 5B-C, Video S7). Interestingly, while loss-of-function mutations in the division machinery (e.g., fts $N-\triangle S P O R, \triangle e n v C$ ) result in an increased cell length, hyperactivation of the division machinery (e.g., ftsL ${ }^{*}$ ) leads to shorter cells than wt (Fig. S9A). Concomitantly, mutants displaying reduced sidewall $P G$ incorporation rates and fewer directionally moving MreB filaments such as $f t s{ }^{*}$ and $\triangle e n v C$, were also found to display significantly increased cell width (Fig. S9B), which are indicative of reduced Rod complex activity ${ }^{59}$. 
340 Notably, the observed interplay between cell elongation and division appeared to impact the geometry of 341 the division site and the shape of the daughter cell poles (Fig. 5D). The ftsN- $\triangle S P O R$ mutant which 342 elongates more rapidly and constricts slower, displayed an elongated division site area and a shallower 343 OM invagination angle at midcell as compared to wild-type cells (Fig. 5D-G). This altered constriction 344 geometry was also observable in the cryo-electron tomograms of dividing cells and correspondingly gave 345 rise to daughter cells with pointier poles than wild-type (Fig. 5F, H). On the other hand, the rapidly 346 constricting $\mathrm{fts} L^{*}$ mutant formed daughters with relatively blunt cell poles (Fig. $\mathbf{5 F}, \mathbf{H}$ ).

348 We reasoned that the variation in division site and polar geometry among the different strains could be 349 related to the activity of the Rod complex at or near the division site. In this case, mutants that take longer 350 to complete the division process (such as $f t s-\Delta S P O R$ ) would allow for more elongation activity in the 351 curved cell region adjacent to the site of constriction, thereby generating shallower constrictions and 352 elongated poles (Fig. 5E-G). Conversely, mutants that divide rapidly (such as $f t s L^{*}$ ) would have less 353 opportunity to elongate the region around the division site and thus form steep constrictions and blunt 354 poles (Fig. 5E-G). To investigate this possibility, we quantified the number of directionally moving MreB 355 filaments in proximity $(\leq 200 \mathrm{~nm})$ to cell constrictions highlighted by Pal-mCherry foci at mid-cell (Fig. 5B356 C, Video S7). Directionally moving MreB filaments were readily observed to pass through division sites in 357 both early and late pre-divisional cells in all strains tested. Notably, however, the ftsN- $\triangle S P O R$ mutant 358 displayed more MreB tracks at the division site at late stages of division than all other strains, and the fts $L^{*}$ 359 mutant showed the least number of total MreB tracks at the division site (Fig. 5C). Thus, the density of 360 MreB tracks at the division site for these cells correlates well with the degree of constriction site and cell 361 pole elongation observed for the different strains. The outlier was the $\triangle e n v C$ mutant, which had an inverted 362 trend of having fewer directionally moving MreB tracks at early division stages than at later points (Fig. 363 5C). We suspect that this change is due to the defect in SPG splitting, which causes a steep curvature of 364 the inner membrane at early points in division that is likely to be unfavorable for MreB localization ${ }^{60,61}$. 365 However, at later stages when SPG processing eventually allows for slow constriction of the OM, this 366 curvature likely becomes more favorable for MreB localization allowing elongation to occur near the division site to generate a shallow constriction like that of the fts $N-\triangle S P O R$ mutant. Overall, these results not only provide strong support for a competition between the PG biosynthetic machineries involved in cell elongation and division, but they also highlight the potential for this competition to define the morphology of the daughter cell poles. 


\section{Discussion}

373 Understanding the mechanisms regulating bacterial division requires unambiguous visualization of the 374 native cell envelope architecture at division sites. Previously obtained data from EM ${ }^{28,62}, \mathrm{CEMOVIS}^{63,64}$ 375 and cryo-ET ${ }^{17,65}$ of Gram-negative bacteria, lacked a clear view of the SPG material produced by the 376 division machinery. Here, we overcome this limitation by combining cryo-FIB milling with cryo-ET to 377 visualize the division site of $E$. coli, providing 3D visualization of the in situ division site architecture at 378 nanometer resolution. Our data reveal several new ultrastructural features of the Gram-negative cell wall, 379 including a wedge-like formation of SPG that may play an important role in fortifying the septum against 380 osmotic rupture. Integrating these observations with dynamic measurements of sPG synthesis and 381 remodeling in several division mutants led to several new mechanistic insights into the process of cell 382 division and how its interplay with cell elongation can modulate cell shape (Fig. 6).

\section{Architecture of the sPG layer}

385 In tomograms of wild-type cells just starting to constrict, all three envelope layers appeared to be 386 invaginating in concert, and little change in the SPG relative to the sidewall PG was evident. However, cells 387 in advanced stages of division had an increased distance between the IM and OM and formed a partial 388 septum. In NAD-filtered tomograms of these cells, a triangular wedge of what is likely to be sPG is observed 389 at the lagging edge of the septum closest to the tip of the invaginating OM. The wedge thins as it 390 approaches the leading edge of the closing IM, and in this narrow portion of the septum, two dense tracks 391 of material are often discernable that correspond to the PG layers that will eventually fortify the daughter 392 cell poles. In cells defective for sPG processing by the amidases, the sPG wedge structure is more 393 prominent than in wild-type cells and it appears to impede the invagination of the OM. We thus infer that 394 amidases process this structure to allow constriction of the OM. Furthermore, because the sPG wedge is 395 observed in deeply constricted wild-type cells as well as unconstricted amidase activation mutants, we 396 suspect that the structure is dynamic with its lagging edge being degraded as new wedge material is 397 deposited at the leading edge. Such a spatial separation of synthesis and degradation would allow the 398 sPG wedge to move in a treadmill-like fashion ahead of the OM as the septum closes.

400 Premature separation of the daughter cell PG layers before a continuous wall structure is formed between 401 them would create a tear in the PG matrix and ultimately cause osmotic cell lysis. The positioning of the 402 SPG wedge at the lagging edge of the septum suggests that it might function to prevent such a catastrophe by providing a buffer of extra PG material linking the daughter cells. Accordingly, the $f t s L^{*}$ mutant lacks an observable SPG wedge structure and has been shown to have a temperature-sensitive lysis phenotype ${ }^{45,46}$. 
407

408

409

410

411

412

413

414

415

416

417

418

419

420

421

422

423

424

425

426

427

428

429

430

431

432

433

434

435

436

437

438

439

440

441

is that the wedge is likely to be continuously remodeled as cells divide gradually whereas the bridge in $S$. aureus appears to provide a static connection between daughter cells until the end of the division process when lesions in the bridge are created and crack propagation results in extremely rapid cell separation ${ }^{66}$.

The enzymes responsible for creating the sPG wedge remain to be identified, but our results with the fts $L^{*}$ mutant suggest that it is not made by $\mathrm{FtsWI}$ synthase. This mutant is thought to hyperactivate $\mathrm{FtsWl}^{30,35-}$ 38. Therefore, if the wedge were produced by the FtsWl synthase, the $f t s L^{*}$ mutant would be expected to produce a thicker or otherwise larger wedge. Instead, it lacks a wedge altogether, suggesting that enhanced FtsWI activity disrupts biogenesis of the SPG wedge by other synthases. An attractive candidate for this additional synthase is the class A penicillin-binding protein (aPBP) PBP1b. Inactivation of PBP1b has been found to be synthetically lethal with defects in FtsWl activation, and the affected mutants were found to lyse due to septal lesions, suggesting that this aPBP promotes division site stability ${ }^{35,67}$. The location of the wedge at the lagging edge of the division site closest to the OM is also consistent with a role for PBP1b in its construction given that this enzyme and related aPBPs require activation by lipoproteins anchored in the $\mathrm{OM}$ to make $\mathrm{PG}^{68,69}$. Thus, the outer fork of the division site where the wedge is located is the only place where aPBPs would be predicted to be functional. Although further work will be required to test this model, it provides an attractive explanation for the division of labor between the aPBP and FtsWI synthases at the site of cell constriction, with the FtsWI synthase promoting ingrowth of the PG layer and the aPBPs providing backfill to counter the activity of SPG remodeling enzymes, thereby stabilizing the septum and preventing lysis.

\section{The sPG activation loop}

The proposal that Fts $\mathrm{N}$ and the amidase enzymes cooperate in a positive feedback loop that promotes SPG synthesis is based on insightful reasoning aimed at explaining how the different domains of FtsN might work together ${ }^{39}$. However, this model lacks direct experimental support. A major prediction of the model is that proper SPG synthesis should require both the SPOR domain of FtsN to recognize denuded glycan strands at the division site and amidase activity to generate them ${ }^{39}$. Our results indicate that this is indeed the case. Both the ftsN- $\triangle S P O R$ mutant and a mutant defective for amidase activation were found to synthesize SPG at a reduced rate compared to wild-type cells. Notably, SPG is still made in these mutants, and even cells defective for all amidases or amidase activators can complete septum formation in E. coli. In the case of the ftsN- $\triangle S P O R$ mutant, other SPOR domain containing division proteins like DedD are likely to be promoting feedback loops analogous to that of $\mathrm{FtsN}^{70}$. However, the observation that mutants defective for all cell division amidases still make septa suggests that although efficient sPG biogenesis depends on the sPG loop, it is not absolutely required to make sPG. 
In addition to stimulating SPG synthesis, the sPG activation pathway in which the SPG loop operates also appears to be important for normal septal architecture. The $f t s L^{*}$ mutant hyperactivates the FtsWI synthase and eliminates the strict FtsN requirement for SPG biogenesis ${ }^{37}$. This short-circuiting of the normal division activation pathway not only causes the loss of the SPG wedge structure observed in wild-type and other mutant cells, but it also promotes the aberrant accumulation of PG within the developing cell poles. Whether this accumulation results from inappropriate activation of PG synthesis by FtsWI or PBP1b, the improper turnover of the deposited material, or some combination of the two remains unknown. Nevertheless, what is clear is that bypassing the normal controls involved in SPG activation and speeding up the process has adverse consequences on the envelope architecture of the poles that are formed. We therefore infer that the normal divisome activation pathway serves an important function in coordinating different activities of the machinery to ensure that division is successfully completed once it is initiated and that the polar end products it produces have a uniform surface.

\section{PG hydrolysis and the Z-ring}

Over the years, many factors have been identified that help to position the Z-ring and condense it at midcell. These proteins are typically thought to act upstream of divisome maturation and the activation of SPG biogenesis ${ }^{6}$. However, our results have uncovered an unexpected connection between the activation of sPG processing by the amidase and Z-ring structure, suggesting there is feedback to the Z-ring from events downstream of SPG synthesis activation. Z-rings were found to be poorly condensed in mutant cells lacking the amidase activator EnvC. Additionally, closely spaced constrictions or areas of sPG biogenesis were also observed at an elevated frequency in these cells, suggesting that division sites are unstable and fail before they complete the division process. Notably, closely spaced constrictions were also evident in prior EM of chaining cells lacking all amidases ${ }^{42}$, and amidase activity has been shown to be critical for the completion of cell constriction in the related Gram-negative bacterium Pseudomonas aeruginosa ${ }^{71}$. Taken together, these results suggest the counterintuitive notion that SPG degradation by the amidases is required to stabilize the divisome, most likely via a positive influence on the structure of the Z-ring that promotes or maintains its condensation. Given that the amidases act on SPG in the periplasm, they are unlikely to directly modulate FtsZ activity. Rather, their effect is probably mediated through SPOR domain proteins like FtsN and DedD that bind the amidase processed glycans ${ }^{40,41}$. These proteins have transmembrane domains and N-terminal cytoplasmic tails, which in the case of FtsN is known to associate with the FtsZ-binding protein $\mathrm{FtsA}^{72,73}$. Thus, the status of SPG biogenesis in the periplasm could be communicated to the Z-ring in the cytoplasm using the binding of SPOR domain proteins to denuded glycans as a proxy. Whether the effect might be mediated simply by concentrating the cytoplasmic domains of SPOR proteins at the division site to modulate the activity of FtsZ-binding proteins or via more complex mechanisms requires further investigation, but the general picture that emerges is that the divisome 
477

478

479

480

481

482

483

484

485

486

487

488

489

490

491

492

493

494

495

496

497

activation pathway is not a one-way street from Z-ring formation to SPG synthesis and processing. The Zring is also likely to be receiving stabilizing/activating signals back from the PG biogenesis machinery.

\section{Cell shape and the balance between cell division and elongation}

The idea that the cell elongation and division machineries may be in competition with one another has been discussed in the field for some time ${ }^{54,55}$. However, it has only been recently that evidence for such a completion has been presented. Cells with a temperature-sensitive allele of fts/ (fts/23) were found to elongate faster than wild-type during growth at the permissive temperature ${ }^{74}$. Conversely, hyperactivated for the Rod complex were found to be longer than a wild-type control, suggesting that division occurs less frequently when cell elongation is stimulated ${ }^{75}$. Finally, the overproduction of cell wall endopeptidases implicated in cell elongation was found to cause lethal cell division defects in mutants impaired for FtsWI activation $^{67}$. Although these findings are supportive of cell elongation occurring at the expense of division and vice versa, it has remained unclear whether this competition occurs at the level of PG synthesis. Here, we used three independent assays (NAM/FDAA incorporation, single-cell elongation rate, and MreB tracking) to demonstrate that septal and side wall PG synthesis rates are inversely correlated to each other, providing strong support for antagonism between the activities of the elongation and division systems which most likely stems from a competition for the limited supply of the lipid II PG precursor. Importantly, our results indicate that this competition does not just affect cell width or how long or short cells are. It also influences the geometry of the septum and the shape of the daughter cell poles. Thus, modulation of the relative activities of the elongation and division systems is likely to play an important role in the generating the diversity of shapes observed among different bacteria. 


\section{Acknowledgments}

499 We are grateful to Phat Vinh Dip, Edward Brignole and Anna Osherov at the MIT.nano cryo-EM facility and 500 KangKang Song and Chen Xu at the University of Massachusetts cryo-EM facility for providing access to 501 the cryo-EM microscopes and for all their help, advice, and maintenance of cryo-EM equipment. 502 Furthermore, we would like to express our gratitude to the MicRoN imaging core at Harvard Medical 503 School, for excellent advice on live cell imaging and maintenance of fluorescence microscopes. We would 504 also like to thank Calixto Saenz and the Microfabrication Core facility at the department of Systems Biology 505 at Harvard Medical School, for the micro-pillar design, fabrication, and consultations. Lastly, we are grateful 506 to Thomas Bartlett and Erkin Kuru for advice and helpful discussions on FDAA and NAM labeling 507 experiments. P.P.N. was supported by the Swiss National Science Foundation (SNF) with both Early 508 Postdoc.Mobility P2BSP3_188112 and Postdoc.Mobility fellowships P400PB_199252. A.V. was supported 509 by a EMBO long-term postdoctoral fellowship ALTF_89-2019 and a SNF Postdoc.Mobility fellowship 510 P500PB_203143. C.A. is funded by Charles University with a PRIMUS grant (PRIMUS/20/SCI/015). This 511 work was also supported by funding from the National Institutes of Health (R35GM142553 to L.H.C. and 512 R01Al083365 to T.G.B.) and Investigator funds from the Howard Hughes Medical Institute (T.G.B.).

\section{Author Contributions}

515 P.P.N and A.V. conceived the project, performed experiments, and analyzed and interpreted the data. 516 P.P.N performed cryo-FIB / cryo-ET and image processing. A.V. generated mutants and performed 517 fluorescence microscopy experiments. V.Y.A. performed 3D segmentations of cryo-ET data. P.M.L. 518 established SIM-TIRF workflow and assisted in data collection. C.A. contributed to cell morphology 519 analyses. L.H.C and T.G.B provided infrastructure and scientific advice. P.P.N, A.V., L.H.C. and T.G.B. 520 wrote the manuscript with input from all authors.

\section{Declaration of Interests}

523 The authors declare that there are no competing financial interests. 


\section{METHODS}

Media, bacterial strains, and mutagenesis

Indicated strain derivatives of E. coli MG1655 used in this study are listed in Table S2-S3. Bacteria were grown in LB (1\% Tryptone, $0.5 \%$ yeast extract, $0.5 \% \mathrm{NaCl}$ ) or $\mathrm{M} 9$ medium ${ }^{76}$ supplemented with $0.2 \% \mathrm{D}$ glucose and casamino acids. For selection, antibiotics were used at $10 \mu \mathrm{g} \mathrm{ml}^{-1}$ (tetracycline), $25 \mu \mathrm{g} \mathrm{ml}$ (chloramphenicol), and 50 (kanamycin, ampicillin) $\mu \mathrm{g} \mathrm{ml}^{-1}$. Mutant alleles were moved between strains using phage $\mathrm{P} 1$ transduction. If necessary, the antibiotic cassette was removed using FLP recombinase expressed from $\mathrm{pCP} 2 \mathrm{O}^{77}$. All mutagenesis procedures were confirmed by PCR.

\section{Cryo-EM specimen preparation}

Figure S1 summarizes the cryo-FIB / cryo-ET pipeline utilized in this study. Bacterial strains were grown overnight in LB media, back diluted $1: 1000$ and incubated shaking at $37^{\circ} \mathrm{C}, 250 \mathrm{rpm}$ to $\mathrm{OD}_{600}=0.3$. Cells were harvested by centrifugation ( $2 \mathrm{~min}, 5000 \times \mathrm{g}, \mathrm{RT}$ ) and resuspended in LB media to a final $\mathrm{OD}_{600}=$ 0.6. Three $\mu \mathrm{L}$ of cell culture were applied to Cflat-2/1 200 mesh copper or gold grids (Electron Microscopy Sciences) glow discharged for 30 seconds at $15 \mathrm{~mA}$. Grids were plunge-frozen in liquid ethane ${ }^{78}$ with a FEI Vitrobot Mark IV (Thermo Fisher Scientific) at RT, 100\% humidity with a waiting time of 10 seconds, one-side blotting time of 13 seconds and blotting force of 10. Customized parafilm sheets were used for one-side blotting. All subsequent grid handling and transfers were performed in liquid nitrogen. Grids were clipped onto cryo-FIB autogrids (Thermo Fisher Scientific).

\section{Cryo-FIB milling}

Grids were loaded in Aquilos 2 Cryo-FIB (Thermo Fisher Scientific). Specimen was sputter coated inside the cryo-FIB chamber with inorganic platinum, and an integrated gas injection system (GIS) was used to deposit an organometallic platinum layer to protect the specimen surface and avoid uneven thinning of cells. Cryo-FIB milling was performed on the specimen using two rectangular patterns to mill top and bottom parts of cells, and two extra rectangular patterns were used to create micro-expansion joints to improve lamellae instability ${ }^{79}$. Cryo-FIB milling was performed at a nominal tilt angle of $14^{\circ}-18^{\circ}$ which translates into a milling angle of $7^{\circ}-11^{\circ 80}$. Cryo-FIB milling was performed in several steps of decreasing ion beam currents ranging from $0.5 \mathrm{nA}$ to $10 \mathrm{pA}$ and decreasing thickness to obtain $100-200 \mathrm{~nm}$ lamellae.

\section{Cryo-electron tomography}

All imaging was done on a FEI Titan Krios (Thermo Fisher Scientific) transmission electron microscope operated at $300 \mathrm{KeV}$ equipped with a Gatan BioQuantum K3 energy filter (20 eV zero-loss filtering) and a Gatan K3 direct electron detector. Prior to data acquisition, a full K3 gain reference was acquired, and ZLP and BioQuantum energy filter were finely tuned. The nominal magnification for data collection was of $42,000 \mathrm{x}$ or $33,000 \mathrm{x}$, giving a calibrated $4 \mathrm{~K}$ pixel size of $2.193 \AA$ and $2.565 / 2.758 \AA$, respectively. Data 
560 collection was performed in the nanoprobe mode using the SerialEM ${ }^{81}$ or Thermo Scientific Tomography

5615.3 software. The tilt range varied depending on the lamella, but generally was from $-70^{\circ}$ to $70^{\circ}$ in $2^{\circ}$ steps

562 following the dose-symmetric tilt scheme ${ }^{82}$. Tilt images were acquired as $8 \mathrm{~K} \times 11 \mathrm{~K}$ super-resolution movies

563 of 4-8 frames with a set dose rate of $1.5-3 \mathrm{e}-/ \mathrm{A} / \mathrm{sec}$. Tilt series were collected at a range of nominal defoci

564 between -3.5 and $-5.0 \mu \mathrm{m}$ and a target total dose of 80 to $180 \mathrm{e}^{-} / \AA^{2}$ (Table S1).

\section{Cryo-electron tomography image processing}

567 Acquired tilted super-resolution movies were motion corrected and Fourier cropped to $4 \mathrm{~K} \times 5 \mathrm{~K}$ stacks, 568 minimizing aliasing effects using framealign from IMOD ${ }^{83}$. Tilt series were aligned using etomo in IMOD ${ }^{84}$ 569 and Dynamo. CTF-estimation was performed in IMOD and/or using customized MATLAB scripts. CTF570 correction was performed by ctfphaseflip program in IMOD ${ }^{85}$. CTF-corrected unbinned tomograms were 571 reconstructed by weighted back projection with and without a SIRT-like filter and subsequently $2 x, 4 x$ and $5728 x$ binned in IMOD ${ }^{84}$.

573 Bandpass filtering and summed projection of cryo-tomogram slices was performed in Dynamo ${ }^{86-89}$ 574 complemented with customized MATLAB scripts. Gaussian and NAD-filtering were performed in Amira 575 (Thermo Fisher Scientific) for visualization purposes. NAD-filtering was applied using the command 576 'Anisotropic Diffusion' in 3D mode for 5 iterations. Gaussian filtering was done by applying the command 577 'Gaussian Filter' under 3D mode with a Kernel size factor of 3. Whole 3D volume FFT filtering was 578 performed in IMOD.

579 Three-dimensional pole curvature rendering was performed in Amira by applying the commands 580 'Curvature' based on the triangulated 3D mesh and 'Shape Index' as implemented in Amira ${ }^{90}$. Shape index 581 (SI) computes the surface scalar field which values are equal to

$$
S I=\frac{\pi}{2} \operatorname{atan} \frac{C_{1}+C_{2}}{C_{1}+C_{2}}
$$

where $C_{1}$ and $C_{2}$ are the two principal curvatures. Shape index ranges from -1 to 1 , negative values indicate negative curvature, positive values indicate positive curvature and values close to 0 indicates flatness of the surface ${ }^{90}$ (Fig. 5).

586

\section{Quantification of cryo-ET data}

588 Division site dimensions

589 Summed projection images of cryo-ET tomograms were used to measure cell dimensions at the division 590 site. Measurements were performed in Fiji1 ${ }^{1}$ using the 'point to point' measuring tool. Measurements were 591 from IM to IM and from OM-OM. Descriptive statistics indicating $\mathrm{N}$ and mean \pm SEM can be found in Fig. 592 S2.

593 Periplasmic space 
Measurements of periplasmic space thickness were performed from OM to IM in the cell areas referred to here as 'side wall', 'pole' and 'curve'. Measurements from IM to IM were performed in the cell area defined in this study as septum (S5 and S8). We used a customized macro in Fiji that measures thirty Euclidean distances from surface-to-surface areas in nm, e.g., from IM to IM at the septum. For these thirty single measurements the mean was calculated, yielding a final single mean value per defined subcellular localization, e.g., septum.

600

Sample preparation for live cell imaging

602 Overnight cultures of indicated E. coli strains were grown in LB supplemented with appropriate antibiotics 603 at $37^{\circ} \mathrm{C}$. The next day, cells were harvested by centrifugation ( $2 \mathrm{~min}, 5000 \mathrm{x} \mathrm{g}, \mathrm{RT}$ ) and washed $2 \times$ with 604 M9 medium. Day cultures were back diluted (1:1000) and grown in M9 (0.2\% D-glucose, casamino acids) 605 supplemented with $50 \mu \mathrm{M}$ IPTG and appropriate antibiotics at $30^{\circ} \mathrm{C}$ until $\mathrm{OD}_{600}=0.2-0.4$. Cells were 606 harvested ( $2 \mathrm{~min}, 5000 \times \mathrm{g}, \mathrm{RT}$ ) and resuspended in $1 / 10^{\text {th }}$ of the original volume. Two microliters of this 607 cell suspension were added onto a $1 \%(\mathrm{w} / \mathrm{v})$ agarose in M9 (0.2\% D-glucose, casamino acids) pad supplemented with $50 \mu \mathrm{M}$ IPTG and covered with a \#1.5 coverslip.

Live cell imaging

611 All samples were imaged on a Nikon Ti-E inverted widefield microscope equipped with a fully motorized 612 stage and perfect focus system. Images were acquired using a 1.45 NA Plan Apo 100x Ph3 DM objective 613 lens with Cargille Type 37 immersion oil. Fluorescence was excited using a Lumencore SpectraX LED light 614 engine and filtered using ET-GFP (Chroma \#49002) and ET-mCherry (Chroma \#49008) filter sets. Images 615 were recorded on an Andor Zyla 4.2 Plus sCMOS camera (65 nm pixel size) using Nikon Elements (v5.10) 616 acquisition software. For subsequent deconvolution procedures, three $200 \mathrm{~nm}$ spaced Z-planes were 617 acquired for both fluorescence channels using 100\% LED output power and 50 ms exposure. Temperature 618 was maintained at $30^{\circ} \mathrm{C}$ using a custom-made environmental enclosure. After a 20 min acclimatization 619 period, cells were imaged at a $2.5 \mathrm{~min}$ acquisition frame rate for a total observation time of 1-4 $\mathrm{h}$.

Image processing for fluorescence microscopy First, time-lapse series and Z-stacks were drift corrected using a customized StackReg plugin in Fiji ${ }^{91,92}$. 623 Subsequently, fluorescence images were deconvolved using the classical maximum likelihood estimation 624 (CMLE) algorithm in Huygens Essential v19.10 (SVI) employing an experimentally derived PSF from 100 $625 \mathrm{~nm}$ TetraSpeck beads (Thermofisher). Image reconstruction was performed over 50 iterations with a 626 quality threshold of 0.01 and a signal-to-noise ratio set to 20 for live-cell imaging and 40 for fluorescent cell wall probes in fixed samples. Chromatic aberrations between different fluorescent wavelengths were postcorrected using the Chromatic Aberration Corrector in Huygens from TetraSpeck bead template. The same 
compared to each other. Last, reconstructed fluorescence images were merged back to phase contrast images and rendered for figure or movie display with Fiji.

Measuring cell envelope constriction dynamics

634 Fluorescent fusions to IM anchored protein ZipA, and OM lipoprotein Pal, allowed us to determine the respective position of the different cell envelope layers during division. Constriction dynamics of IM and OM were derived from kymographs generated using the Fiji plugin KymographClear ${ }^{93}$ and automictically split into forward and reverse trajectories using Fourier filtering. This filtering step allows us to measure the constriction rate for each side independently. Constriction kinetics were derived by automatically extracting the fluorescent trajectories for ZipA and Pal using KymographDirect ${ }^{93}$. Anisotropy of the division process was determined by taking the ratio of the constriction velocity between the forward and reverse trajectory. Only cells where the division site displayed no signs of displacement except for constriction were analyzed to eliminate confounding effects on the analysis by excessive cell movement (e.g., pushing).

Measuring division site circularity of vertically imaged cells were applied. Briefly, a silicon wafer containing $5.5 \mu \mathrm{m}$ long and $1.5 \mu \mathrm{m}$ wide photo-resist pillars was generated following high aspect ratio photolithography procedures with an adhesion layer. The dimension of these pillars reaches the practically feasible aspect ratio for photolithography designs and thus impedes increasing pillar length without concomitantly increasing width, precluding elongated or chaining division mutants. A modified silanization surface treatment with plasma cleaning was applied to increase the surface hydrophobicity of the silicon wafer to minimize agarose accumulation. Agarose micro holes were generated by pouring degassed $6 \%$ agarose (w/v) in $\mathrm{H}_{2} \mathrm{O}$ on the silicon wafer. Agarose was allowed to solidify for $40 \mathrm{~min}$ at RT and was peeled of, cut into $5 \times 5 \mathrm{~mm}$ pieces, and incubated in M9 medium supplemented with $0.2 \%$ D-glucose, casamino acids, $25 \mu \mathrm{g} \mathrm{ml}^{-1}$ chloramphenicol and $50 \mu \mathrm{m} \mathrm{ml}^{-1}$ ampicillin overnight.

Cells were grown as described for sample preparation for live-cell imaging added on agarose pads. Cells which were not trapped in micro holes were washed off gently using $1 \mathrm{ml}$ of growth medium. Five micrometer spanning Z-stacks (at a $200 \mathrm{~nm}$ step size) were acquired and subsequently deconvolved. were segmented using Laplacian algorithm in combination the peripheral fluorescence setting. Circularity $(C)$ is calculated in Morphometrics as:

$$
C=\frac{P^{2}}{4 \Pi \times A^{2}}
$$


665

666

667

668

669

670

671

672

673

674

675

676

677

678

679

680

681

682

683

684

685

686

687

688

689

690

691

692

693

694

695

696

697

698

699

where $(P)$ is the perimeter and $(A)$ is the area enclosed by the circle and is a dimensionless measure. A perfect circle displays a circularity of 1 , while increasing values correspond to less circular objects.

Measuring Z-ring condensation from time-lapse data.

Condensation of cytoskeletal elements was addressed using similar procedures described by Squyres et al., 2021. Briefly, five frames (corresponding to $10 \mathrm{~min}$ ) from recorded time-lapse series were sumprojected in Fiji. Z-rings in these sum projection images were then aligned along the length axis and average-intensity projected into single image. Fluorescence intensity was measured across the full width along horizontal axis of the averaged projection image. Intensity values were normalized and their corresponding FWHM values were calculated in MATLAB.

\section{Measuring Z-ring condensation from 3D data.}

Similar procedures as outline for measuring Z-ring condensation in time-lapse series were applied. Two micrometer spanning Z-stacks (at a $200 \mathrm{~nm}$ step size) were acquired to capture a full three-dimensional view of a cell. Images were restored in Huygens as described above. Image volumes were sum-projected into a single plane, Z-rings extracted, aligned, and averaged described above. Fluorescence intensity profiles were measured identically as for time-lapse data. Snapshots for three-dimensional maximum intensity projections were rendered in Huygens.

\section{Measuring cell wall synthesis rates by biorthogonal NAM probes.}

Septal cell wall synthesis rates were measured as described previously here ${ }^{98,99}$. NAM-Alkyne was purchased as a custom synthesis product from Tocris following the procedures of Liang et al., 2017. All experiments were carried out in $\triangle$ murQ background and in presence of pCF $436^{100}$ for ITPG inducible expression of AmgK and MurU. Overnight cultures were back diluted 1:1000 into fresh LB containing 15 $\mu \mathrm{g} \mathrm{ml}{ }^{-1}$ gentamycin. Cells were grown at $37^{\circ} \mathrm{C}$ until $\mathrm{OD}_{600}=0.4$. Subsequently, $1.5 \mathrm{ml}$ of cells were harvested ( $2 \mathrm{~min}, 5000 \mathrm{xg}, \mathrm{RT}$ ) and resuspended in $300 \mu \mathrm{LB}$ containing $1 \mathrm{mM}$ IPTG and $0.5 \mathrm{mM}$ HADA to label all cell wall material by FDAAs. Samples were incubated rotating at $37^{\circ} \mathrm{C}$ for $30 \mathrm{~min}$. Endogenous UDP-NAM production was inhibited by the addition of $200 \mu \mathrm{g} \mathrm{ml}^{-1}$ fosfomycin. After 10 min incubation, cells were washed twice in $1.5 \mathrm{ml} \mathrm{LB}, 1 \mathrm{mM} \mathrm{IPTG}, 200 \mathrm{\mu g} \mathrm{ml}^{-1}$ fosfomycin. Next, cells were incubated for $15 \mathrm{~min}$ in the presence of $0.2 \%(\mathrm{w} / \mathrm{v})$ NAM-Alkyne, $1 \mathrm{mM} \mathrm{IPTG}, 200 \mu \mathrm{g} \mathrm{ml}^{-1}$ fosfomycin at $37^{\circ} \mathrm{C}$. Cells were fixed using ice-cold $70 \%(\mathrm{w} / \mathrm{v})$ ethanol for $20 \mathrm{~min}$ at $4^{\circ} \mathrm{C}$. Next, cells pellets were washed $3 \mathrm{x}$ with $1 \mathrm{x}$ PBS. Biorthogonal NAM-Alkyne probes were labeled by click chemistry using $5 \mu \mathrm{M}$ of Alexa488 azide substrate according to manufactures instruction. Samples were stored in $20 \mu \mathrm{lPBS}$ at $4^{\circ} \mathrm{C}$ and imaged within $48 \mathrm{~h}$ of labeling experiment. 
700

701

702

703

704

705

706

707

708

709

710

711

712

713

714

715

716

717

718

719

720

721

722

723

724

725

726

727

728

729

730

731

732

733

734

735

Samples were imaged on Nikon Ti2-E inverted widefield microscope equipped with a Lumencor Spectra III light engine, and Semrock dichroics (LED-CFP/YFP/mCherry-3X-A-000, LED-DA/FI/TR/Cy5/Cy7-5X-A000) and emission filters (FF01-432/36, FF01-515/30, FF01-544/24). Images were recorded using a 1.45 NA Plan Apo 100 x PH3 oil objective with Olympus Type F immersion oil and a pco.edge 4.2bi Back Illuminated Cooled sCMOS camera using Nikon Elements 5.2.

One micrometer spanning Z-stacks (separated by $200 \mathrm{~nm}$ ) were acquired and subsequently deconvolved as described under image processing for fluorescence microscopy. Z-stacks were sum-projected using Fiji. De novo septal PG synthesis was measured by integrating the fluorescence intensity of NAM-Alexa488 along the division site using the line tool (width $=3$ pixels). Levels of cell wall hydrolysis were assessed by measuring the overall reduction in HADA fluorescence as compared to baseline signal intensity derived from fixing cells prior to NAM chase.

Measuring cell wall remodeling by FDAA incorporation.

For FDAA pulse chase experiments, cells grown overnight were back diluted 1:1000 in fresh LB and grown until OD600 $=0.4$ at $37^{\circ} \mathrm{C}$. Subsequently, $1.5 \mathrm{ml}$ of cells were harvested $(2 \mathrm{~min}, 5000 \times \mathrm{g}$, RT) and resuspended in $300 \mu \mathrm{LB}$ containing $0.5 \mathrm{mM}$ YADA. Samples were incubated rotating at $37^{\circ} \mathrm{C}$ for $40 \mathrm{~min}$. Cells were washed once in $1.5 \mathrm{ml} \mathrm{LB}$ and resuspended in $300 \mu \mathrm{LB}$ containing $0.5 \mathrm{mM}$ HADA. Samples were incubated at $37^{\circ} \mathrm{C}$ for either for $2 \mathrm{~min}, 4 \mathrm{~min}$, and $8 \mathrm{~min}$ prior to immediate fixation with $70 \%$ ethanol. After fixation, cells were washed $3 \mathrm{x}$ in PBS, stored in the dark at $4^{\circ} \mathrm{C}$ and imaged within $48 \mathrm{~h}$. The same image acquisition and analyses procedures were carried out as described for NAM probes. In addition to the division site, fluorescence intensities measurements were also performed along the side wall and polar region of the cells.

\section{Cell shape quantification analyses}

Bacterial cells were segmented and analyzed from still phase contrast images or time-lapse series using the software package Morphometrics ${ }^{96}$. Results from Morphometrics were post-processed using customized MATLAB scripts to exclude erroneously segmented cell debris in live image data based on area. Cell width, length and pole angles per segmented cell were directly extracted from Morphometrics. We obtained the invagination angle from both sides of the cell at the invagination site. The invagination site is defined as the narrowest segment of the cell, e.g., lowest cell width value, that present negative curvature on both sides of the cell body. The cell elongation $(E)$ was determined by a customized MATLAB script:

$$
E=L_{i}-L_{0}
$$

where $L$ is cell length, $L_{0}$ is the cell length in the first frame and $\mathrm{i}=1, \ldots, I$, where $I$ is the number of frames. The elongation rate is the slope of the linear regression performed on the plotted elongation values over time in Prism (GraphPad 9.0.0). 


\section{SIM-TIRF Microscopy and MreB Tracking}

Samples were prepared as described for live-cell imaging. Cells were added to high precision \# 1.5 coverslips (Marienfeld) and placed on a $1 \%(\mathrm{w} / \mathrm{v})$ agarose pad in M9 (0.2\% D-glucose, casamino acids) and imaged at room temperature on a Nikon Ti-2 N-SIM microscope, equipped with N-SIM spatial light modulator illuminator, TIRF Lun-F laser combiner with 488 and $561 \mathrm{~nm}$ laser lines, a N-SIM 488/561 dual band dichroic mirror, SR HP Apo TIRF 100x 1.5 NA oil objective with automated correction collar and Hamamatsu Orca Flash 4.0 camera attached to a Cairn Research Twimcam splitter with a ET525/50m or a ET605/70m emission filter (for MreB-sw-mNeonGreen or Pal-mCherry fusion, respectively). The refractive index of the immersion oil (1.512) (GE Heatlhcare) was optimized for MreB-sw-mNeonGreen signal and corrected using the automated correction collar for the Pal-mCherry fusion. Alignment of the 488 and 561 lasers for SIM-TIRF and 3D-SIM and of the N-SIM optics and illumination was performed before each experiment at the image plane. First, a $3 \mathrm{~min}$ time-lapse series (at $3 \mathrm{~s}$ acquisition frame rate) in SIM-TRIF mode was collected using 20 \% laser power with 100 ms exposure time to follow MreB-swmNeonGreen dynamics. Then, a single slice of a 3D-SIM Pal-mCherry (40 \% laser power, $100 \mathrm{~ms}$ exposure) and a brightfield reference image was acquired. Raw fluorescence images were reconstructed using Nikon Elements 5.11 acquisition Software with indicated settings: MreB illumination contrast 0.8, noise suppression 0.3 and blur suppression 0.05; Pal illumination contrast 3.75, noise suppression 0.1 and blur suppression 0.5 . Only reconstructed images with a quality score $\geq 8$ and passed SIMcheck quality test $^{101}$ were used for further analysis. Subsequently MreB time-lapse series was overlayed over the reference channels in Fiji.

Particle tracking was performed in Fiji using the TrackMate v6.0.1 plugin ${ }^{102}$. MreB filaments were detected using the LoG-detector with an estimated radius of $0.3 \mu \mathrm{m}$. Spurious spots were filtered using a quality threshold of 50. Spots were linked using a Kalman filter with an initial search radius for $0.2 \mu \mathrm{m}$ and search radius for $0.1 \mu \mathrm{m}$. No frame gaps were allowed. Only Tracks consisting of $\geq 4$ continuous spots (12 s) and traveled less than $1 \mu \mathrm{m}$ in total distance were kept for further analysis. To analyze the nature of the displacement of each track, the mean square displacement $(M S D)$ was calculated using the MATLAB class msdanalyzer ${ }^{103}$ following the equation:

$$
\operatorname{MSD}(\tau)=2 d D \tau
$$

where $(t)$ is the delay time and $(D)$ is the diffusion coefficient. Slopes $(\alpha)$ of the individual MSD curves were extracted using the Log-log fit of the MSD and the delay time $\tau$. As the maximum delay time $75 \%$ of the track length was used. Tracks with a $R^{2}$ for $\log [M S D]$ versus $\log [t]$ below 0.95 indicative of a poor fit to the MSD curve were excluded from the analysis. MreB filaments engaged in active cell wall synthesis are displaced by the enzymatic action of the enzymatic activities of RodA and PBP $2 \mathrm{~b}^{56-58}$ and thus it's MSD curves display slopes of $\alpha \approx 2$ indicative of a transported particle motion above the rate of Brownian diffusion (Fig. S10B). MreB filaments in constricting cells, as determined by the presence of a Pal-mCherry 
772 foci at the division site, were analyzed by fitting a $200 \mathrm{~nm}$ wide region of interest to the cell division site.

773 Directional MreB tracks were deemed to contribute to the elongation of the division site. Early and late 774 division stages were distinguished by the presence of two separated Pal foci or a continuous fluorescent 775 signal across the cell, respectively.

776

777

\section{Statistical analysis}

778 All data measurements were plotted and analyzed using GraphPad Prism 9 (Version 9.1.2). In general, 779 (log-) normal distribution was tested by using Shapiro-Wilk test, for comparisons of two groups, significance 780 was determined by two-tailed, unpaired Student's $t$ test with Welch correction and $F$ test for variance 781 analysis. One-way ANOVA test were used for comparison of more than two groups using the 782 recommended post-test for selected pairwise comparisons. All experiments were carried out at least with 7833 independent biological replicates. $P$ values less than 0.05 were considered statistically significant. Levels 784 of significance are indicated by ${ }^{*} p<0.05,{ }^{* *} p<0.01,{ }^{* * *} p<0.001,{ }^{* * *} p<0.0001$; ns, not significant. 


\section{References}

803

804

805

806

807

808

809

810

811

812

813

814

815

816

817

818

819

820

821

822

823

824

825

826

827

828

829

830

831

832

1. Silhavy, T. J., Kahne, D. \& Walker, S. The bacterial cell envelope. Cold Spring Harb. Perspect. Biol. 2, a000414 (2010).

2. Konovalova, A., Kahne, D. E. \& Silhavy, T. J. Outer Membrane Biogenesis. Annu. Rev. Microbiol. 71, 539-556 (2017).

3. Rohs, P. D. A. \& Bernhardt, T. G. Growth and Division of the Peptidoglycan Matrix. Annu. Rev. Microbiol. (2021) doi:10.1146/annurev-micro-020518-120056.

4. Du, S. \& Lutkenhaus, J. At the Heart of Bacterial Cytokinesis: The Z Ring. Trends Microbiol. 27, 781791 (2019).

5. Mahone, C. R. \& Goley, E. D. Bacterial cell division at a glance. J. Cell Sci. 133, (2020).

6. Adams, D. W. \& Errington, J. Bacterial cell division: assembly, maintenance and disassembly of the Z ring. Nat. Rev. Microbiol. 7, 642-653 (2009).

7. Beveridge, T. J. Ultrastructure, Chemistry, and Function of the Bacterial Wall. in International Review of Cytology (eds. Bourne, G. H., Danielli, J. F. \& Jeon, K. W.) vol. 72 229-317 (Academic Press, 1981).

8. Erickson, H. P. How bacterial cell division might cheat turgor pressure - a unified mechanism of septal division in Gram-positive and Gram-negative bacteria. BioEssays 39, 1700045 (2017).

9. Matias, V. R. F. \& Beveridge, T. J. Native Cell Wall Organization Shown by Cryo-Electron Microscopy Confirms the Existence of a Periplasmic Space in Staphylococcus aureus. J. Bacteriol. 188, 1011-1021 (2006).

10. Matias, V. R. F. \& Beveridge, T. J. Cryo-electron microscopy of cell division in Staphylococcus aureus reveals a mid-zone between nascent cross walls. Mol. Microbiol. 64, 195-206 (2007).

11. Khanna, K. et al. The molecular architecture of engulfment during Bacillus subtilis sporulation. eLife 8, e45257 (2019).

12. Khanna, K., Lopez-Garrido, J., Sugie, J., Pogliano, K. \& Villa, E. Asymmetric localization of the cell division machinery during Bacillus subtilis sporulation. eLife 10, e62204 (2021).

13. Lopez-Garrido, J. et al. Chromosome Translocation Inflates Bacillus Forespores and Impacts Cellular Morphology. Cell 172, 758-770.e14 (2018).

14. Nanninga, N., Koppes, L. J. H. \& de Vries-Tijssen, F. C. The cell cycle of Bacillus subtilis as studied by electron microscopy. Arch. Microbiol. 123, 173-181 (1979).

15. Burdett, I. D. \& Murray, R. G. Septum formation in Escherichia coli: characterization of septal structure and the effects of antibiotics on cell division. J. Bacteriol. 119, 303-324 (1974).

16. Judd, E. M. et al. Distinct Constrictive Processes, Separated in Time and Space, Divide Caulobacter Inner and Outer Membranes. J. Bacteriol. 187, 6874-6882 (2005).

17. Szwedziak, P., Wang, Q., Bharat, T. A. M., Tsim, M. \& Löwe, J. Architecture of the ring formed by the tubulin homologue FtsZ in bacterial cell division. eLife 3, e04601 (2014).

18. Frank, J. Electron Tomography: Methods for Three-Dimensional Visualization of Structures in the Cell. (Springer Science \& Business Media, 2008).

19. Grimm, R., Typke, D., Bärmann, M. \& Baumeister, W. Determination of the inelastic mean free path in ice by examination of tilted vesicles and automated most probable loss imaging. Ultramicroscopy 63, 169-179 (1996).

20. Oikonomou, C. M. \& Jensen, G. J. Cellular Electron Cryotomography: Toward Structural Biology In Situ. Annu. Rev. Biochem. 86, 873-896 (2017).

21. Hayles, M. F. et al. The making of frozen-hydrated, vitreous lamellas from cells for cryo-electron microscopy. J. Struct. Biol. 172, 180-190 (2010).

22. Marko, M., Hsieh, C., Schalek, R., Frank, J. \& Mannella, C. Focused-ion-beam thinning of frozenhydrated biological specimens for cryo-electron microscopy. Nat. Methods 4, 215-217 (2007). 
833

834

835

836

837

838

839

840

841

842

843

844

845

846

847

848

849

850

851

852

853

854

855

856

857

858

859

860

861

862

863

864

865

866

867

868

869

870

871

872

873

874

875

876

877

878

23. Medeiros, J. M. et al. Robust workflow and instrumentation for cryo-focused ion beam milling of samples for electron cryotomography. Ultramicroscopy 190, 1-11 (2018).

24. Rigort, A. et al. Focused ion beam micromachining of eukaryotic cells for cryoelectron tomography. Proc. Natl. Acad. Sci. 109, 4449-4454 (2012).

25. Villa, E., Schaffer, M., Plitzko, J. M. \& Baumeister, W. Opening windows into the cell: focused-ionbeam milling for cryo-electron tomography. Curr. Opin. Struct. Biol. 23, 771-777 (2013).

26. de Winter, D. A. M. et al. In-situ integrity control of frozen-hydrated, vitreous lamellas prepared by the cryo-focused ion beam-scanning electron microscope. J. Struct. Biol. 183, 11-18 (2013).

27. Zhang, J., Ji, G., Huang, X., Xu, W. \& Sun, F. An improved cryo-FIB method for fabrication of frozen hydrated lamella. J. Struct. Biol. 194, 218-223 (2016).

28. Burdett, I. D. \& Murray, R. G. Electron microscope study of septum formation in Escherichia coli strains B and B-r during synchronous growth. J. Bacteriol. 119, 1039-1056 (1974).

29. Berezuk, A. M., Goodyear, M. \& Khursigara, C. M. Site-directed Fluorescence Labeling Reveals a Revised N-terminal Membrane Topology and Functional Periplasmic Residues in the Escherichia coli Cell Division Protein FtsK. J. Biol. Chem. 289, 23287-23301 (2014).

30. Yang, X. et al. A two-track model for the spatiotemporal coordination of bacterial septal cell wall synthesis revealed by single-molecule imaging of FtsW. Nat. Microbiol. 6, 584-593 (2021).

31. Burman, L. G. \& Park, J. T. Molecular model for elongation of the murein sacculus of Escherichia coli. Proc. Natl. Acad. Sci. U. S. A. 81, 1844-1848 (1984).

32. Taguchi, A. et al. FtsW is a peptidoglycan polymerase that is functional only in complex with its cognate penicillin-binding protein. Nat. Microbiol. 4, 587-594 (2019).

33. Weiss, D. S. et al. Localization of the Escherichia coli cell division protein FtsI (PBP3) to the division site and cell pole. Mol. Microbiol. 25, 671-681 (1997).

34. Du, S. \& Lutkenhaus, J. Assembly and Activation of the Escherichia coli Divisome. Mol. Microbiol. 105, 177-187 (2017).

35. Liu, B., Persons, L., Lee, L. \& Boer, P. A. J. de. Roles for both FtsA and the FtsBLQ subcomplex in FtsN-stimulated cell constriction in Escherichia coli. Mol. Microbiol. 95, 945-970 (2015).

36. Park, K.-T., Du, S. \& Lutkenhaus, J. Essential Role for FtsL in Activation of Septal Peptidoglycan Synthesis. mBio 11, e03012-20.

37. Tsang, M.-J. \& Bernhardt, T. G. A role for the FtsQLB complex in cytokinetic ring activation revealed by an ftsL allele that accelerates division. Mol. Microbiol. 95, 925-944 (2015).

38. Marmont, L. S. \& Bernhardt, T. G. A conserved subcomplex within the bacterial cytokinetic ring activates cell wall synthesis by the FtsW-Ftsl synthase. Proc. Natl. Acad. Sci. U. S. A. 117, 2387923885 (2020).

39. Gerding, M. A. et al. Self-Enhanced Accumulation of FtsN at Division Sites and Roles for Other Proteins with a SPOR Domain (DamX, DedD, and RlpA) in Escherichia coli Cell Constriction. J. Bacteriol. 191, 7383-7401 (2009).

40. Ursinus, A. et al. Murein (peptidoglycan) binding property of the essential cell division protein Fts $\mathrm{N}$ from Escherichia coli. J. Bacteriol. 186, 6728-6737 (2004).

41. Yahashiri, A., Jorgenson, M. A. \& Weiss, D. S. Bacterial SPOR domains are recruited to septal peptidoglycan by binding to glycan strands that lack stem peptides. Proc. Natl. Acad. Sci. U. S. A. 112, 11347-11352 (2015).

42. Heidrich, C. et al. Involvement of $\mathrm{N}$-acetylmuramyl-L-alanine amidases in cell separation and antibiotic-induced autolysis of Escherichia coli. Mol. Microbiol. 41, 167-178 (2001).

43. Uehara, T., Parzych, K. R., Dinh, T. \& Bernhardt, T. G. Daughter cell separation is controlled by cytokinetic ring-activated cell wall hydrolysis. EMBO J. 29, 1412-1422 (2010). 
879

880

881

882

883

884

885

886

887

888

889

890

891

892

893

894

895

896

897

898

899

900

901

902

903

904

905

906

907

908

909

910

911

912

913

914

915

916

917

918

919

920

921

922

923

924

44. Uehara, T., Dinh, T. \& Bernhardt, T. G. LytM-domain factors are required for daughter cell separation and rapid ampicillin-induced lysis in Escherichia coli. J. Bacteriol. 191, 5094-5107 (2009).

45. Ishino, F. et al. New mutations fts-36, Its-33, and ftsW clustered in the mra region of the Escherichia coli chromosome induce thermosensitive cell growth and division. J. Bacteriol. 171, 5523-5530 (1989).

46. Ueki, M., Wachi, M., Jung, H. K., Ishino, F. \& Matsuhashi, M. Escherichia coli mraR gene involved in cell growth and division. J. Bacteriol. 174, 7841-7843 (1992).

47. Kuru, E. et al. In Situ Probing of Newly Synthesized Peptidoglycan in Live Bacteria with Fluorescent D-Amino Acids. Angew. Chem. Int. Ed. 51, 12519-12523 (2012).

48. Hsu, Y.-P., Booher, G., Egan, A., Vollmer, W. \& VanNieuwenhze, M. S. d-Amino Acid Derivatives as in Situ Probes for Visualizing Bacterial Peptidoglycan Biosynthesis. Acc. Chem. Res. 52, 27132722 (2019).

49. Bartlett, T. M. et al. A Periplasmic Polymer Curves Vibrio cholerae and Promotes Pathogenesis. Cell 168, 172-185.e15 (2017).

50. Liang, H. et al. Metabolic labelling of the carbohydrate core in bacterial peptidoglycan and its applications. Nat. Commun. 8, 15015 (2017).

51. Hale, C. A. \& de Boer, P. A. Direct binding of FtsZ to ZipA, an essential component of the septal ring structure that mediates cell division in E. coli. Cell 88, 175-185 (1997).

52. Buss, J. et al. In vivo organization of the FtsZ-ring by ZapA and ZapB revealed by quantitative superresolution microscopy. Mol. Microbiol. 89, 1099-1120 (2013).

53. Squyres, G. R. et al. Single-molecule imaging reveals that Z-ring condensation is essential for cell division in Bacillus subtilis. Nat. Microbiol. 6, 553-562 (2021).

54. Begg, K. J. et al. The balance between different peptidoglycan precursors determines whether Escherichia coli cells will elongate or divide. J. Bacteriol. 172, 6697-6703 (1990).

55. Lleo, M. M., Canepari, P. \& Satta, G. Bacterial cell shape regulation: testing of additional predictions unique to the two-competing-sites model for peptidoglycan assembly and isolation of conditional rod-shaped mutants from some wild-type cocci. J. Bacteriol. 172, 3758-3771 (1990).

56. Domínguez-Escobar, J. et al. Processive movement of MreB-associated cell wall biosynthetic complexes in bacteria. Science 333, 225-228 (2011).

57. Garner, E. C. et al. Coupled, circumferential motions of the cell wall synthesis machinery and MreB filaments in B. subtilis. Science 333, 222-225 (2011).

58. van Teeffelen, S. et al. The bacterial actin MreB rotates, and rotation depends on cell-wall assembly. Proc. Natl. Acad. Sci. U. S. A. 108, 15822-15827 (2011).

59. Dion, M. F. et al. Bacillus subtilis cell diameter is determined by the opposing actions of two distinct cell wall synthetic systems. Nat. Microbiol. 4, 1294-1305 (2019).

60. Hussain, S. et al. MreB filaments align along greatest principal membrane curvature to orient cell wall synthesis. eLife 7, e32471 (2018).

61. Ursell, T. S. et al. Rod-like bacterial shape is maintained by feedback between cell curvature and cytoskeletal localization. Proc. Natl. Acad. Sci. U. S. A. 111, E1025-1034 (2014).

62. Dubochet, J., McDowall, A. W., Menge, B., Schmid, E. N. \& Lickfeld, K. G. Electron microscopy of frozen-hydrated bacteria. J. Bacteriol. 155, 381 (1983).

63. Matias, V. R. F., Al-Amoudi, A., Dubochet, J. \& Beveridge, T. J. Cryo-Transmission Electron Microscopy of Frozen-Hydrated Sections of Escherichia coli and Pseudomonas aeruginosa. J. Bacteriol. 185, 6112-6118 (2003).

64. Zuber, B. et al. Direct visualization of the outer membrane of mycobacteria and corynebacteria in their native state. J. Bacteriol. 190, 5672-5680 (2008). 
925

926

927

928

929

930

931

932

933

934

935

936

937

938

939

940

941

942

943

944

945

946

947

948

949

950

951

952

953

954

955

956

957

958

959

960

961

962

963

964

965

966

967

968

969

970

971

65. Yao, Q. et al. Short FtsZ filaments can drive asymmetric cell envelope constriction at the onset of bacterial cytokinesis. EMBO J. 36, 1577-1589 (2017).

66. Zhou, X. et al. Mechanical crack propagation drives millisecond daughter cell separation in Staphylococcus aureus. Science 348, 574-578 (2015).

67. Truong, T. T., Vettiger, A. \& Bernhardt, T. G. Cell division is antagonized by the activity of peptidoglycan endopeptidases that promote cell elongation. Mol. Microbiol. 114, 966-978 (2020).

68. Paradis-Bleau, C. et al. Lipoprotein cofactors located in the outer membrane activate bacterial cell wall polymerases. Cell 143, 1110-1120 (2010).

69. Typas, A. et al. Regulation of peptidoglycan synthesis by outer-membrane proteins. Cell 143, 10971109 (2010).

70. Liu, B., Hale, C. A., Persons, L., Phillips-Mason, P. J. \& de Boer, P. A. J. Roles of the DedD Protein in Escherichia coli Cell Constriction. J. Bacteriol. 201, e00698-18 (2019).

71. Yakhnina, A. A., McManus, H. R. \& Bernhardt, T. G. The cell wall amidase AmiB is essential for Pseudomonas aeruginosa cell division, drug resistance and viability. Mol. Microbiol. 97, 957-973 (2015).

72. Busiek, K. K. \& Margolin, W. A role for FtsA in SPOR-independent localization of the essential Escherichia coli cell division protein FtsN. Mol. Microbiol. 92, 1212-1226 (2014).

73. Lyu, Z. et al. FtsN activates septal cell wall synthesis by forming a processive complex with the septum-specific peptidoglycan synthase in E. coli. 2021.08.23.457437

https://www.biorxiv.org/content/10.1101/2021.08.23.457437v1 (2021)

doi:10.1101/2021.08.23.457437.

74. Coltharp, C., Buss, J., Plumer, T. M. \& Xiao, J. Defining the rate-limiting processes of bacterial cytokinesis. Proc. Natl. Acad. Sci. 113, E1044-E1053 (2016).

75. Rohs, P. D. A. et al. A central role for PBP2 in the activation of peptidoglycan polymerization by the bacterial cell elongation machinery. PLoS Genet. 14, e1007726 (2018).

76. Miller, J. H. Experiments in molecular genetics. (Cold Spring Harbor Laboratory, 1972).

77. Datsenko, K. A. \& Wanner, B. L. One-step inactivation of chromosomal genes in Escherichia coli K12 using PCR products. Proc. Natl. Acad. Sci. U. S. A. 97, 6640-6645 (2000).

78. Dubochet, J. et al. Cryo-electron microscopy of vitrified specimens. Q. Rev. Biophys. 21, 129-228 (1988).

79. Wolff, G. et al. Mind the gap: Micro-expansion joints drastically decrease the bending of FIB-milled cryo-lamellae. J. Struct. Biol. 208, 107389 (2019).

80. Wagner, F. R. et al. Preparing samples from whole cells using focused-ion-beam milling for cryoelectron tomography. Nat. Protoc. 15, 2041-2070 (2020).

81. Mastronarde, D. N. SerialEM: A Program for Automated Tilt Series Acquisition on Tecnai Microscopes Using Prediction of Specimen Position. Microsc. Microanal. 9, 1182-1183 (2003).

82. Hagen, W. J. H., Wan, W. \& Briggs, J. A. G. Implementation of a cryo-electron tomography tiltscheme optimized for high resolution subtomogram averaging. J. Struct. Biol. 197, 191-198 (2017).

83. Kremer, J. R., Mastronarde, D. N. \& Mclntosh, J. R. Computer visualization of three-dimensional image data using IMOD. J. Struct. Biol. 116, 71-76 (1996).

84. Mastronarde, D. N. \& Held, S. R. Automated tilt series alignment and tomographic reconstruction in IMOD. J. Struct. Biol. 197, 102-113 (2017).

85. Xiong, Q., Morphew, M. K., Schwartz, C. L., Hoenger, A. H. \& Mastronarde, D. N. CTF Determination and Correction for Low Dose Tomographic Tilt Series. J. Struct. Biol. 168, 378-387 (2009).

86. Castaño-Díez, D., Kudryashev, M., Arheit, M. \& Stahlberg, H. Dynamo: A flexible, user-friendly development tool for subtomogram averaging of cryo-EM data in high-performance computing environments. J. Struct. Biol. 178, 139-151 (2012). 
972

973

974

975

976

977

978

979

980

981

982

983

984

985

986

987

988

989

990

991

992

993

994

995

996

997

998

999

000

001

002

.003

004

005

006

007

008

009

010

87. Castaño-Díez, D. The Dynamo package for tomography and subtomogram averaging: components for MATLAB, GPU computing and EC2 Amazon Web Services. Acta Crystallogr. Sect. Struct. Biol. 73, (2017).

88. Castaño-Díez, D., Kudryashev, M. \& Stahlberg, H. Dynamo Catalogue: Geometrical tools and data management for particle picking in subtomogram averaging of cryo-electron tomograms. J. Struct. Biol. 197, 135-144 (2017).

89. Navarro, P. P., Stahlberg, H. \& Castaño-Díez, D. Protocols for Subtomogram Averaging of Membrane Proteins in the Dynamo Software Package. Front. Mol. Biosci. 5, (2018).

90. Koenderink, J. J. \& van Doorn, A. J. Surface shape and curvature scales. Image Vis. Comput. 10, 557-564 (1992).

91. Schindelin, J. et al. Fiji: an open-source platform for biological-image analysis. Nat. Methods 9, 676682 (2012).

92. Ringel, P. D., Hu, D. \& Basler, M. The Role of Type VI Secretion System Effectors in Target Cell Lysis and Subsequent Horizontal Gene Transfer. Cell Rep. 21, 3927-3940 (2017).

93. Mangeol, P., Prevo, B. \& Peterman, E. J. G. KymographClear and KymographDirect: two tools for the automated quantitative analysis of molecular and cellular dynamics using kymographs. Mol. Biol. Cell 27, 1948-1957 (2016).

94. Söderström, B., Chan, H., Shilling, P. J., Skoglund, U. \& Daley, D. O. Spatial separation of FtsZ and FtsN during cell division. Mol. Microbiol. 107, 387-401 (2018).

95. Bisson-Filho, A. W. et al. Treadmilling by FtsZ filaments drives peptidoglycan synthesis and bacterial cell division. Science 355, 739-743 (2017).

96. Ursell, T. et al. Rapid, precise quantification of bacterial cellular dimensions across a genomic-scale knockout library. BMC Biol. 15, 17 (2017).

97. Squyres, G. R. et al. Single-molecule imaging reveals that Z-ring condensation is essential for cell division in Bacillus subtilis. Nat. Microbiol. 6, 553-562 (2021).

98. Liang, H. et al. Metabolic labelling of the carbohydrate core in bacterial peptidoglycan and its applications. Nat. Commun. 8, 15015 (2017).

99. Yang, X. et al. A two-track model for the spatiotemporal coordination of bacterial septal cell wall synthesis revealed by single-molecule imaging of FtsW. Nat. Microbiol. 1-10 (2021) doi:10.1038/s41564-020-00853-0.

100. Fumeaux, C. \& Bernhardt, T. G. Identification of MupP as a New Peptidoglycan Recycling Factor and Antibiotic Resistance Determinant in Pseudomonas aeruginosa. mBio 8, (2017).

101. Ball, G. et al. SIMcheck: a Toolbox for Successful Super-resolution Structured Illumination Microscopy. Sci. Rep. 5, 15915 (2015).

102. Tinevez, J.-Y. et al. TrackMate: An open and extensible platform for single-particle tracking. Methods San Diego Calif 115, 80-90 (2017).

103. Tarantino, N. et al. TNF and IL-1 exhibit distinct ubiquitin requirements for inducing NEMO-IKK supramolecular structures. J. Cell Biol. 204, 231-245 (2014). 


\section{A I. Constriction}

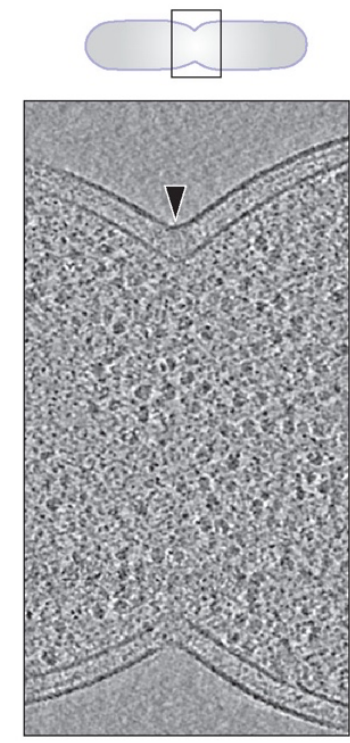

B
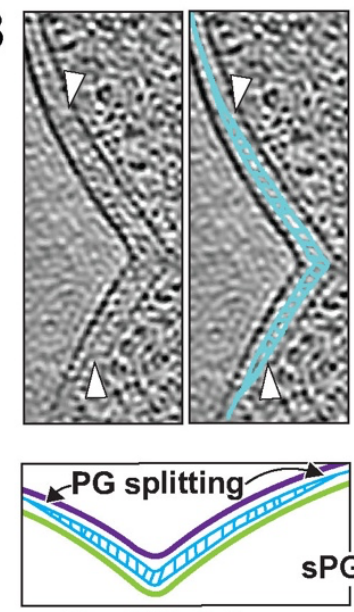

Two PG plates
II. Septation
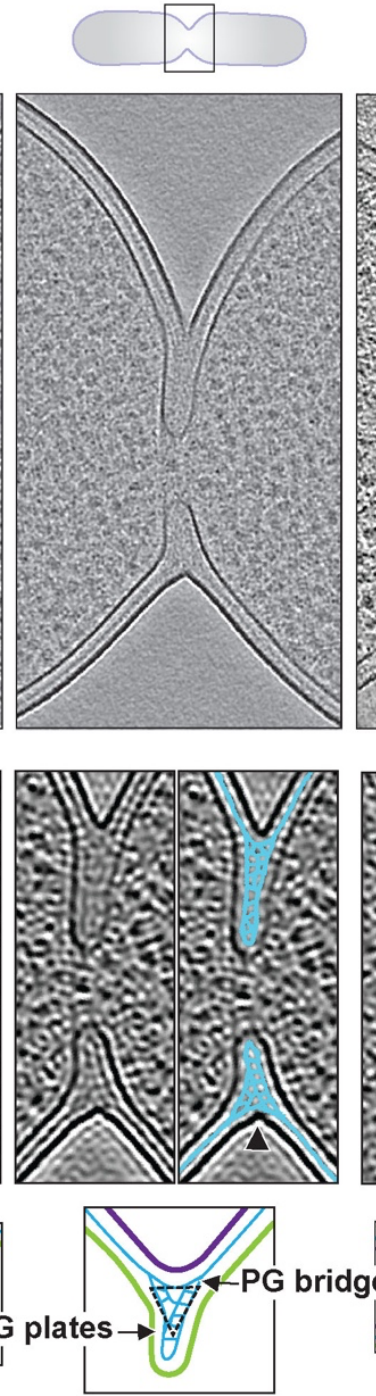

III. Cytokinesis
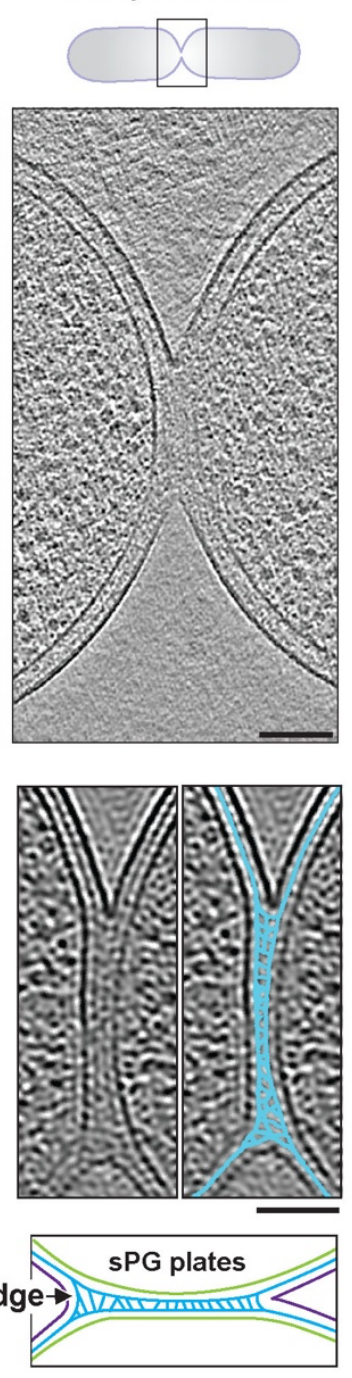
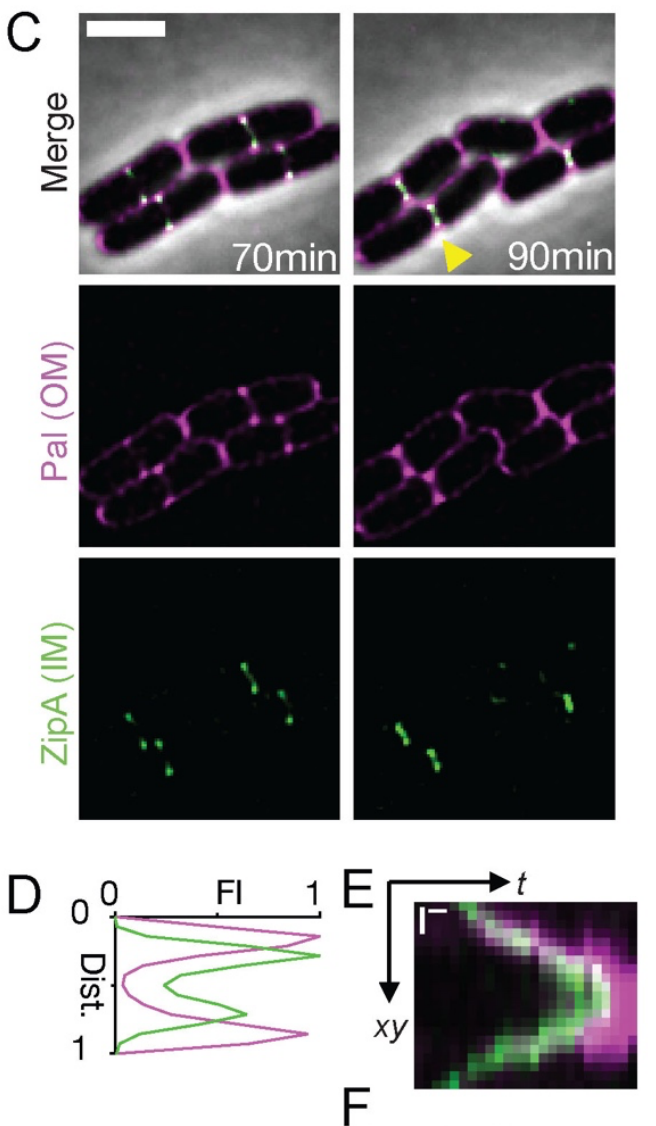

Constriction velocity

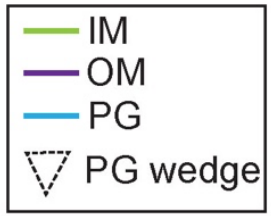

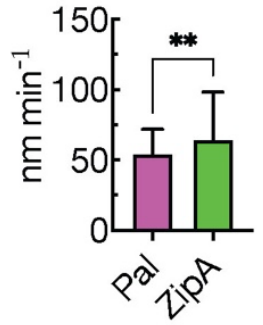

Figure 1: In situ cell envelope architecture and dynamics during $E$. coli cell division. (A) Overview of different stages of cell division. Summed, projected central slices of cryo-electron tomograms visualizing different stages in division of wild-type $E$. coli are shown. Black arrowhead indicates division site side displayed in B. (B) NAD-filtered cryo-electron tomograms visualizing the cell wall. Left panels show a 3D slice, right panels show the corresponding slice with segmented PG signal in cyan (see Methods). White arrowheads indicate where the PG layer appears to thicken from one to two layers, and black arrowhead indicates division site side shown in the schematic overview for the septation stage of the visualized cell envelope architecture. (C) Time-lapse series of wild-type E. coli expressing Pal-mCherry and ZipA-sfGFP as $\mathrm{OM}$ and IM markers, respectively, imaged at $30^{\circ} \mathrm{C}$ on $\mathrm{M} 9$ supplemented with $0.2 \%$ casamino acids and D-glucose. Fluorescence signals were deconvolved (see Methods). Yellow triangles mark division sites used for line scans of fluorescence intensity profiles (D) and kymograph analysis of cytokinesis (E). (F) Constriction velocity of IM and OM were derived from the slopes of the fluorescence signals in kymographs (see Methods). All data are expressed as mean + one SD. Two-sided unpaired t-test; ${ }^{* *} p<0.01 ; N=150$ division kymographs. Scale bar: $(A)$ and $(B)=100 \mathrm{~nm},(C)=2 \mu \mathrm{m}$, and $(E)=200 \mathrm{~nm}$ (vertical); $5 \mathrm{~min}$ (horizontal). 
A
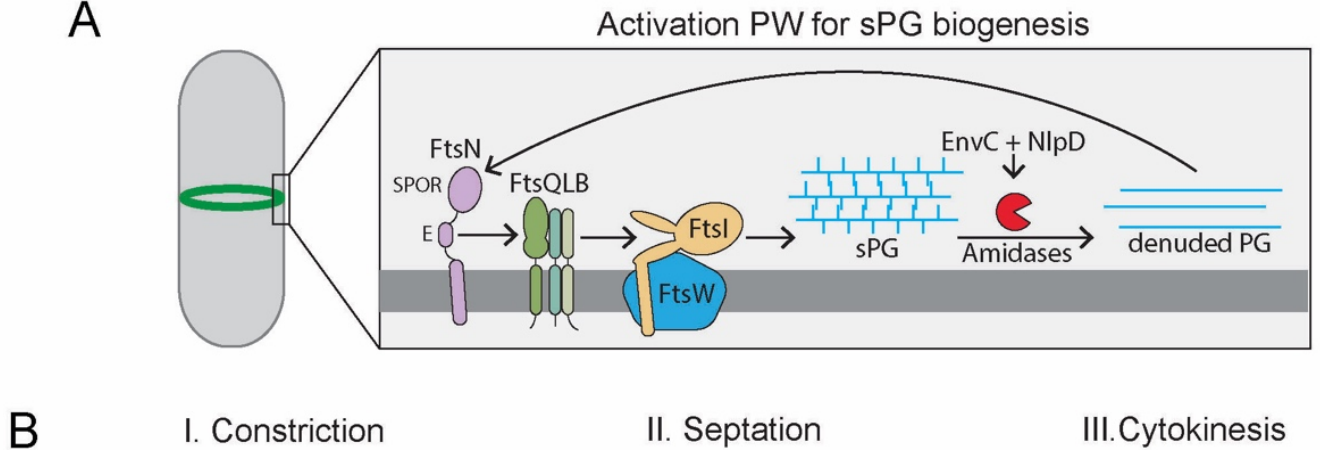

II. Septation

III. Cytokinesis
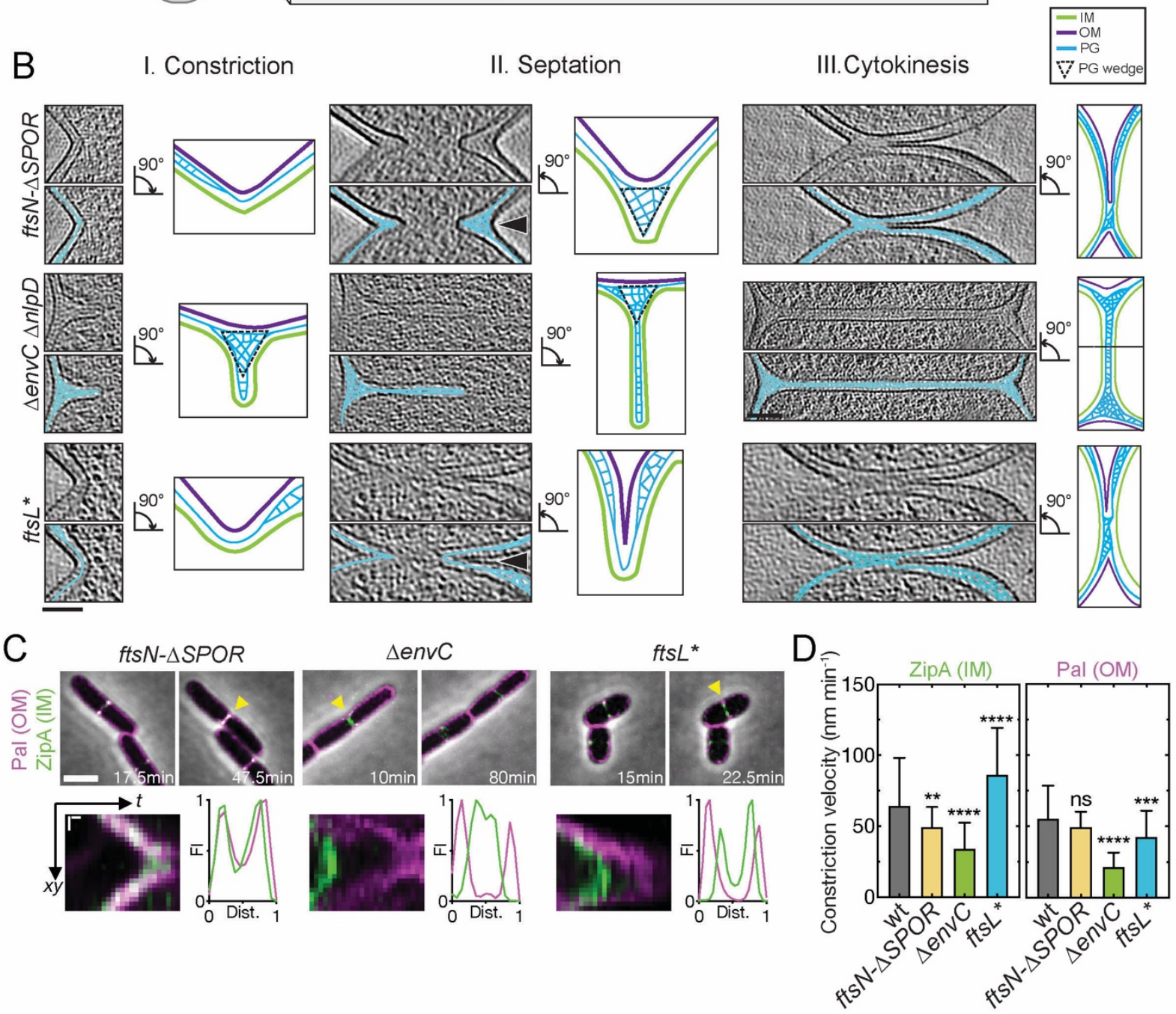

Figure 2: Divisome mutants display altered division site ultrastructure and constriction kinetics. (A) Schematic overview of the septal PG loop pathway for the activation of SPG synthesis (see text for details). (B) NAD-filtered cryo-electron tomograms of division sites in the indicated division mutants of $E$. coli. Top panels show a 3D slice and bottom panels show the corresponding slice with segmented PG signal in cyan (see Methods). Right panels show summary diagrams of the cell envelope architecture visualized by cryo-ET. Black arrowheads indicate division site side represented in the schemes. (C) Timelapse series of indicated E. coli division mutants expressing Pal-mCherry and ZipA-sfGFP as OM and IM markers, respectively, imaged as in Figure 1. Yellow triangles mark division sites used for kymograph analysis and line scans of fluorescence intensity profiles of cytokinesis. (D) Constriction velocity of the IM and OM were determined as in Figure 1. All data are expressed as mean + one SD. Data from wild-type is replotted from Fig. 1F for comparison. Brown-Forsythe and Welch ANOVA tests, differences in significances are tested relative to wild-type; ${ }^{* *} p<0.01,{ }^{* * *}=p<0.001,{ }^{* * *} p<0.0001 ; N=150$ division kymographs for each strain. Scale bar: $(B)=100 \mathrm{~nm}$ and $(C)$ top row $=2 \mu \mathrm{m}$ and bottom row kymographs $=200 \mathrm{~nm}$ (vertical); $5 \mathrm{~min}$ (horizontal). 


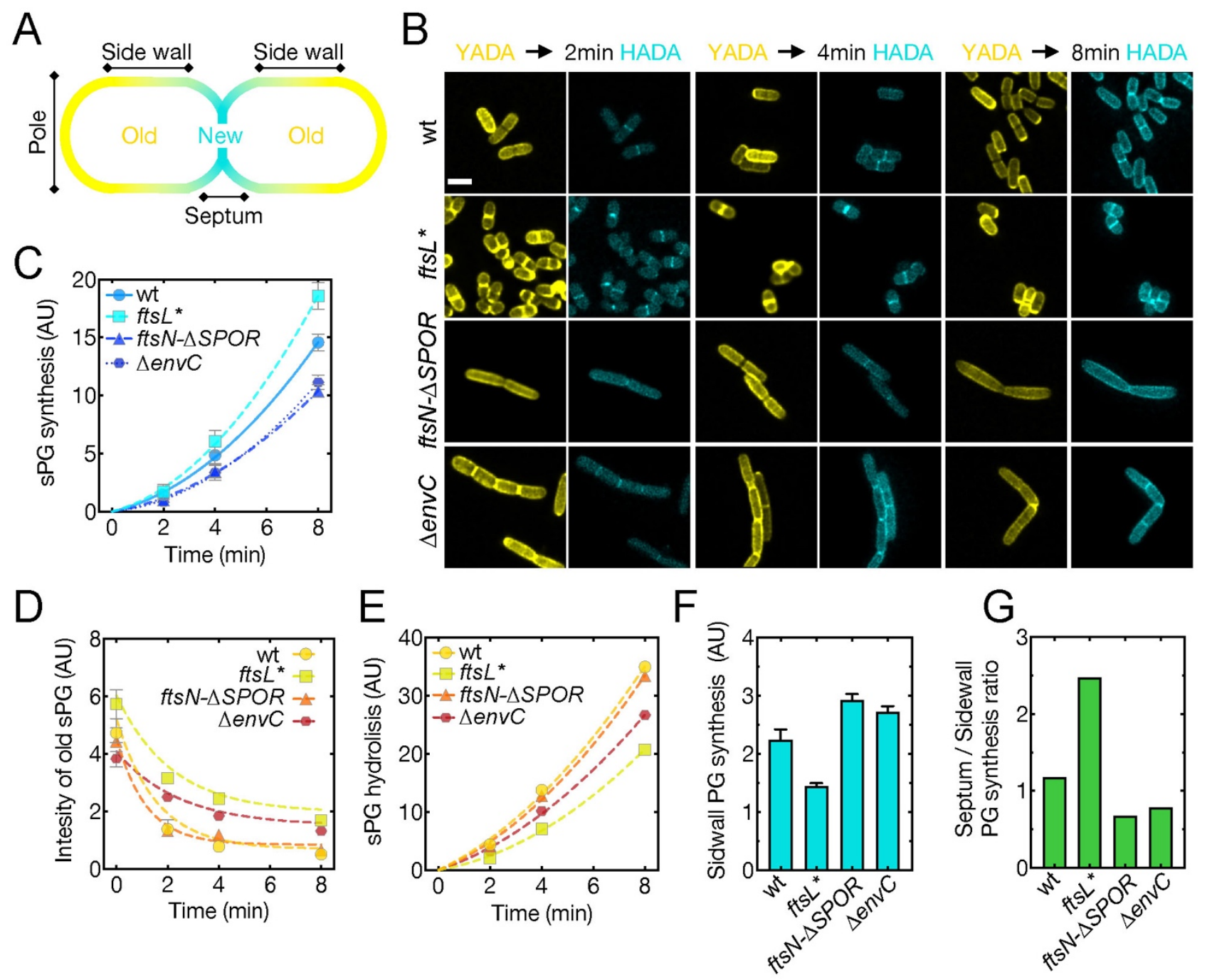

Figure 3: FDAA assay for cell wall synthesis and hydrolysis rates during division and elongation. (A) Schematic representation of labeling patterns observed for the pulse-chase experiment. New cell wall material is labeled with HADA (blue), while old material is stained with YADA (yellow). (B) Representative images of indicated strains after 2, 4 and, 8 min pulses with HADA. Integrated fluorescence intensity was measured and normalized at the division site area for new (C) and old (D) PG. Deriving the integral from these intensity measurements yields the rate of SPG synthesis. Data was fit to a quadratic exponential equation (R-squared $>0.9$ ). (E) Septal $P G$ hydrolysis rates were derived from calculating the reduction in YADA fluorescence intensity over time, as compared to cells fixed prior to chase (time point $0 \mathrm{~min}, \mathrm{D})$. (F) Sidewall incorporation of new cell wall material (HADA fluorescence intensity) was measured after 8 min, due low signal intensities in earlier timepoints. (G) Ratio between sPG and sidewall synthesis was calculated by dividing the mean HADA fluorescence intensity for each after the 8 min pulse. All data are represented as mean fluorescence intensity. Error bars represent $95 \%$ confidence intervals. All values are arbitrary fluorescence intensity units and were divided by 1000 for plotting purposes. Scale bar in (A) $=2 \mu \mathrm{m}$. 


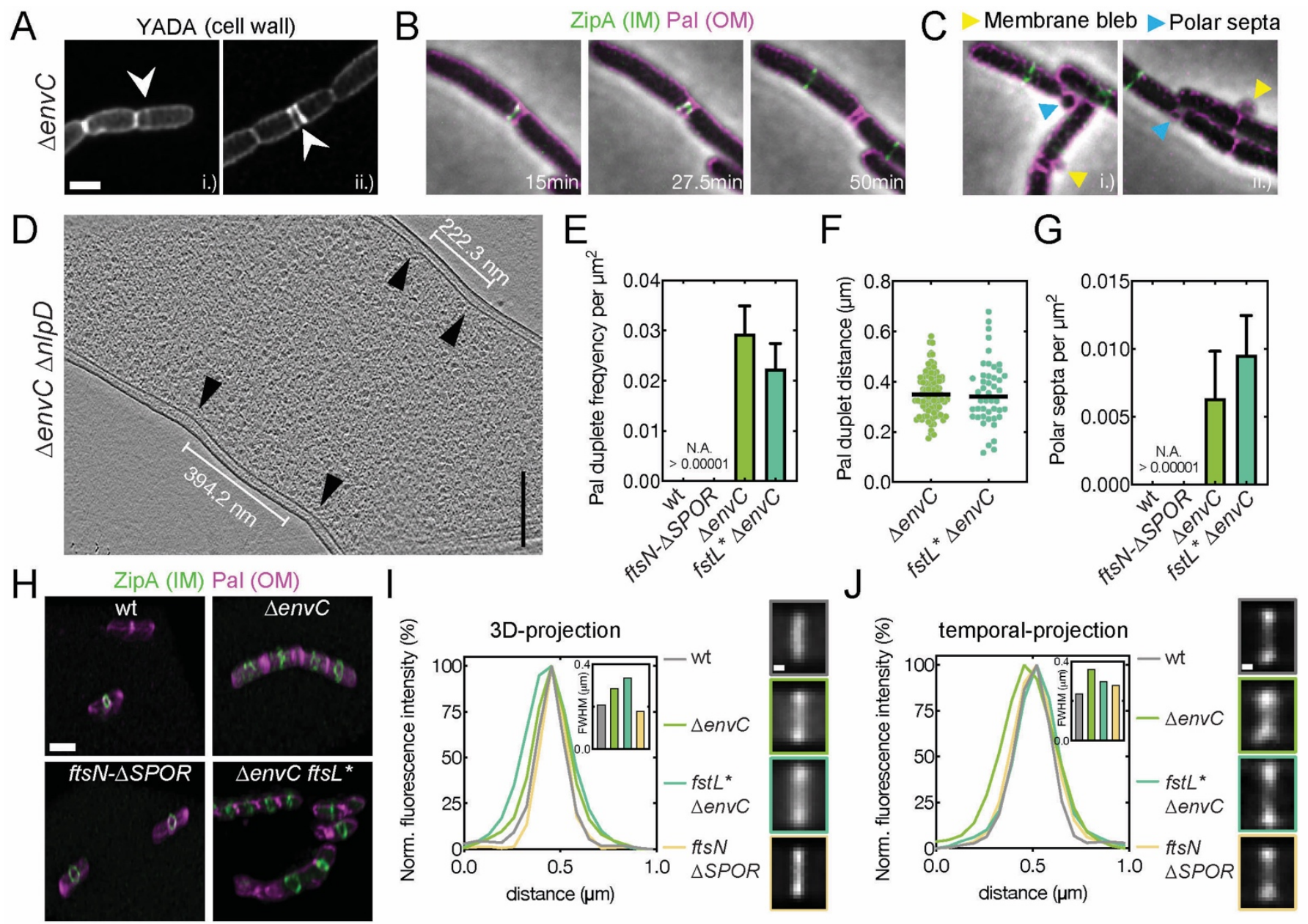

Figure 4: SPG hydrolysis is required for normal Z-ring placement and condensation. (A) Distribution of cell wall material in $\triangle e n v C$ cells was assessed by FDAA staining. Images are sum-projections of a $1 \mu \mathrm{m}$ spanning z-stack and were deconvolved. White arrowheads indicated double septa. (B) Time-lapse series of a $\triangle$ envC mutant expressing Pal-mCherry and ZipA-sfGFP as OM and IM markers, respectively, imaged as in Figure 1. An example of double septum formation is shown. (C) Examples for membrane blebbing (yellow arrowheads) and polar septa (blue arrowheads) formation are highlighted. (D) Formation of double constrictions observed in cryo-electron tomograms of $\triangle e n v C \Delta n l p D$ cells. (E) Frequency of double septum formation was quantified from counting the number of Pal-mCherry doublets and normalizing for cell area. No $P a l$ doublets were found $>10,000$ cells for wild-type or $\mathrm{fts} N-\triangle S P O R$ cells. Data is represented as mean + SD. (F) Distance between Pal duplets was measured manually using the line tool in Fiji. (G) Frequency of polar septa was measured for the indicated strains and normalized per cell area. No polar septa were observed in > 10,000 wild-type or $\mathrm{ftsN}-\triangle S P O R$ cells. $(\mathrm{H})$ Three-dimensional maximum intensity renderings showing Z-ring condensation based on ZipA-sfGFP localization. Scale bar $=2 \mu \mathrm{m}$. The degree of Z-ring condensation was quantified from averaged fluorescence intensity projections from summed 3D volumes (I) or from 5 time points (corresponding to $10 \mathrm{~min}$ ) of a time-lapse series $(\mathrm{J})$. Width of the fluorescence signal distribution across the horizontal axis was used to determine degree of condensation. FWHM is shown as an insert. Averaged Z-rings are shown and color-coded according to graphs. Scale bars: $(A)-(C)$ $=2 \mu \mathrm{m},(\mathrm{D})=200 \mathrm{~nm},(\mathrm{H})=2 \mu \mathrm{m},(\mathrm{I})-(\mathrm{J})=200 \mathrm{~nm}$. 
bioRxiv preprint doi: https://doi.org/10.1101/2021.10.02.462887; this version posted October 3, 2021. The copyright holder for this preprint (which was not certified by peer review) is the author/funder, who has granted bioRxiv a license to display the preprint in perpetuity. It is made available under aCC-BY-NC-ND 4.0 International license.

A

C
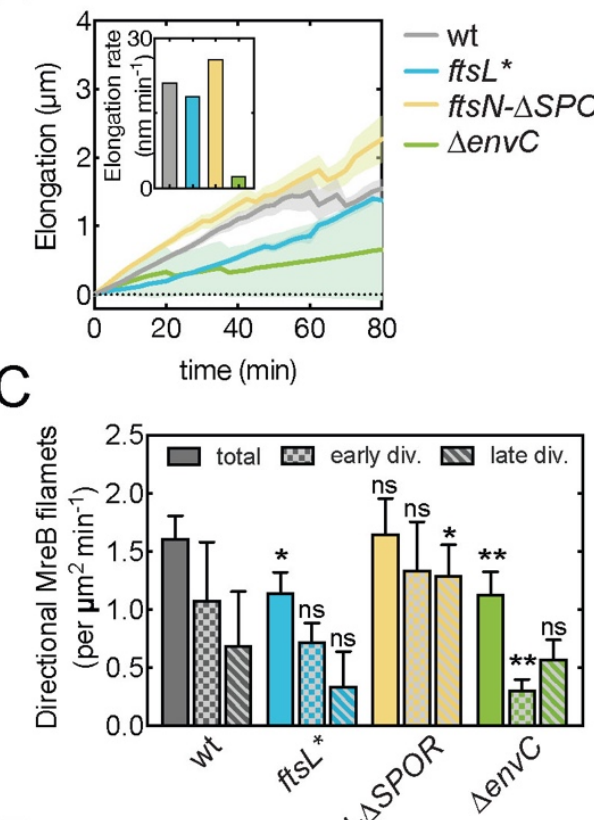

$\mathrm{E}$

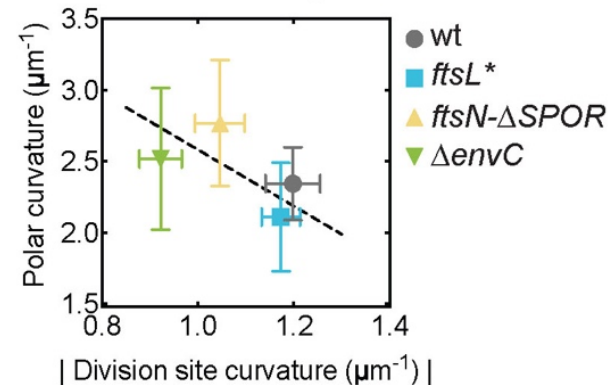

$\mathrm{H}$

$\mathrm{H}$

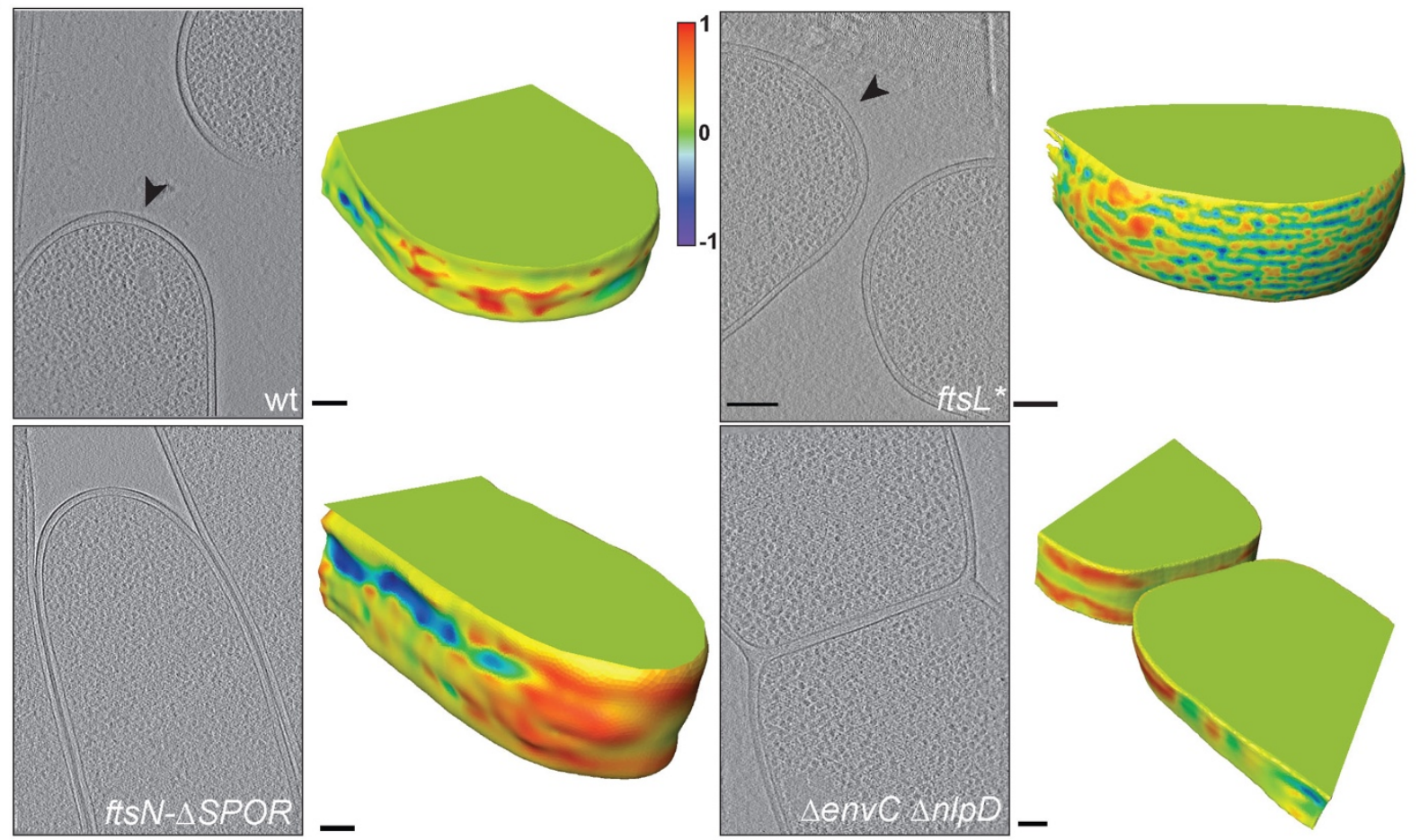

B

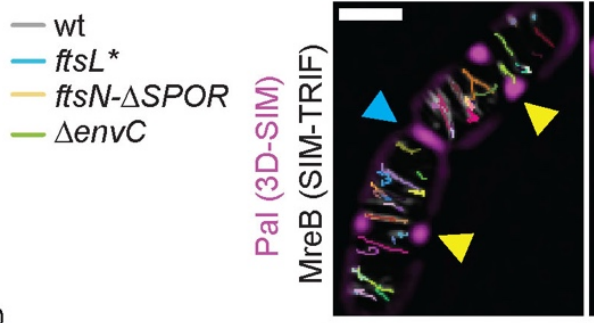

D

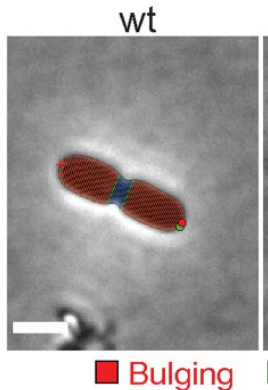

Early divison site
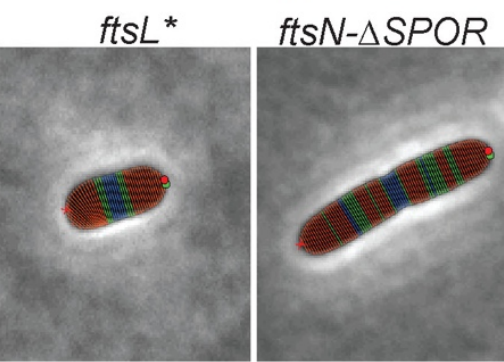

senvC

F

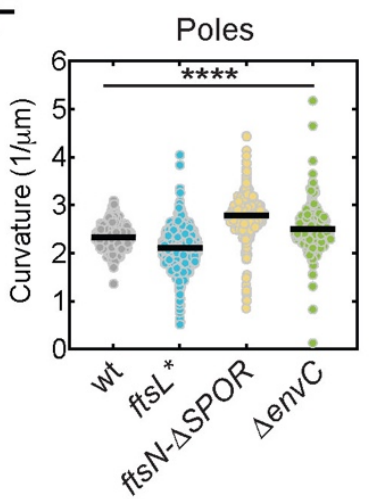

G

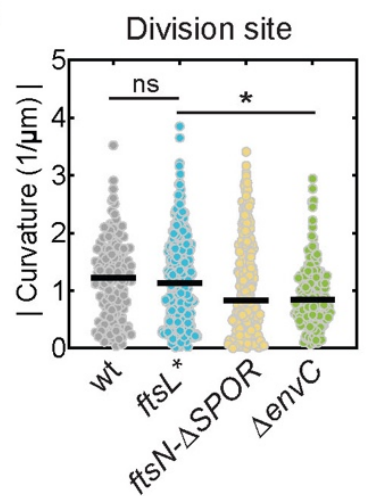

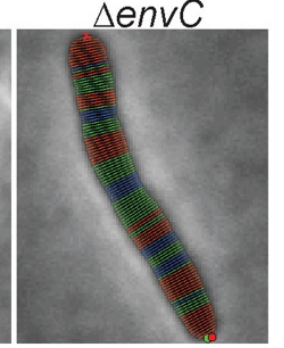

1 x Pole 2

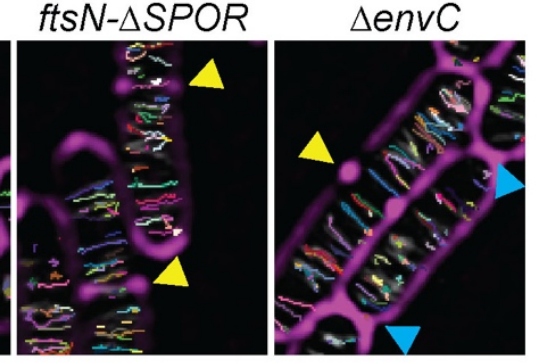

Late divison site 
Figure 5: Competition between the divisome and elongation machinery defines polar cell shape. (A) Single-cell elongation was measured for indicated mutants grown at $30^{\circ} \mathrm{C}$ on $\mathrm{M} 9$ supplemented with $0.2 \%$ casamino acids and D-glucose for $80 \mathrm{~min}$. Elongation rate (insert) was determined from the slope of a best fit linear regression model. (B) MreB dynamics were followed using an mNeonGreen sandwich fusion (MreB ${ }^{\mathrm{sw}-\mathrm{mNG}}$ ) in the indicated strains for $3 \mathrm{~min}$ at 3 second acquisition frame rate using SIM-TIRF microscopy. Time-lapse series were sum projected and overlayed with single particle tracking results from TrackMate and 3D-SIM Pal-mCherry reference images. Pal-mCherry signal serves to identify constricted cells. Early division site (yellow arrowheads) displayed Pal foci which were resolvable as two distinct foci, whereas late division sites (blue arrowheads) displayed a continuous Pal signal across the cell body, indicative of complete or near complete cytokinesis. (C) Directionally moving MreB tracks were filtered by MSD analysis (see Methods) and plotted as mean + SD and normalized by cell area. One-way ANOVA test, differences in significances are tested relative to wild-type for each corresponding stage; * $p<0.05$, ${ }^{*}$ $\mathrm{p}<0.01$, ns $=$ non-significant. (D) Representative phase contrast micrographs showing segmented cells in Morphometrics for the indicated division mutants. Color-coded legend indicates cell regions with positive curvature on both sides (red, bulging), negative curvature on both sides (blue, constricting) and positive curvature on one side while negative curvature on the other side (green, bending). Red dots indicate poles. Scale bar $=2 \mu \mathrm{m}$. (E-G) Polar curvature was measured by the two highest points of positive cell outline curvature, while constriction curvature was assessed by measuring the opposing contour-matched lowest curvature values at the division site using Morphometrics (see Methods). Polar and division site curvatures are negatively correlated. Ordinary One-way ANOVA; ${ }^{*} p<0.05,{ }^{* * * *} p<0.0001$, ns = non-significant. $(H)$ Summed, projected central 3D slices through cryo-electron tomograms visualizing cell poles. Black arrowheads indicate 3D rendered pole. The corresponding 3D-volume renderings show the type of curvature present on the pole surface determined by shape index (see Methods). Summed projection images are scaled. Scale bars: $(B)=1 \mu \mathrm{m},(D)=2 \mu \mathrm{m}(\mathrm{H})$ summed projection images $=200 \mathrm{~nm}$ and 3D renderings $=100 \mathrm{~nm}$. 
A Synthesis $=$ hydrolysis
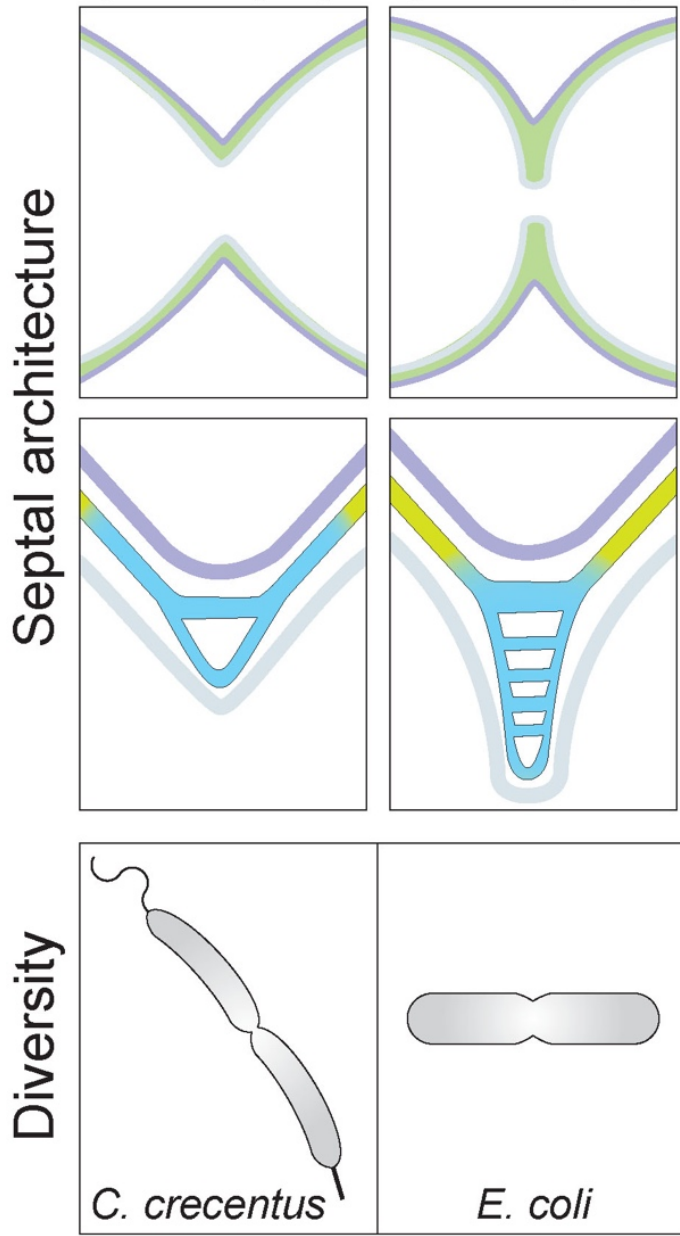

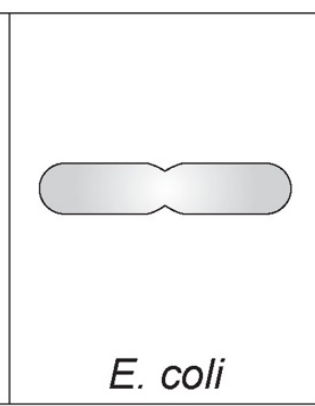

E. coli
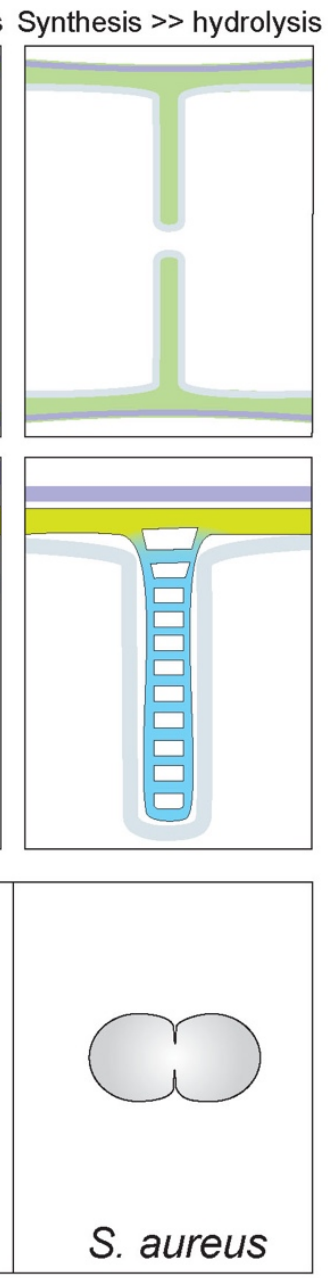

B

\section{PG insertion balance}
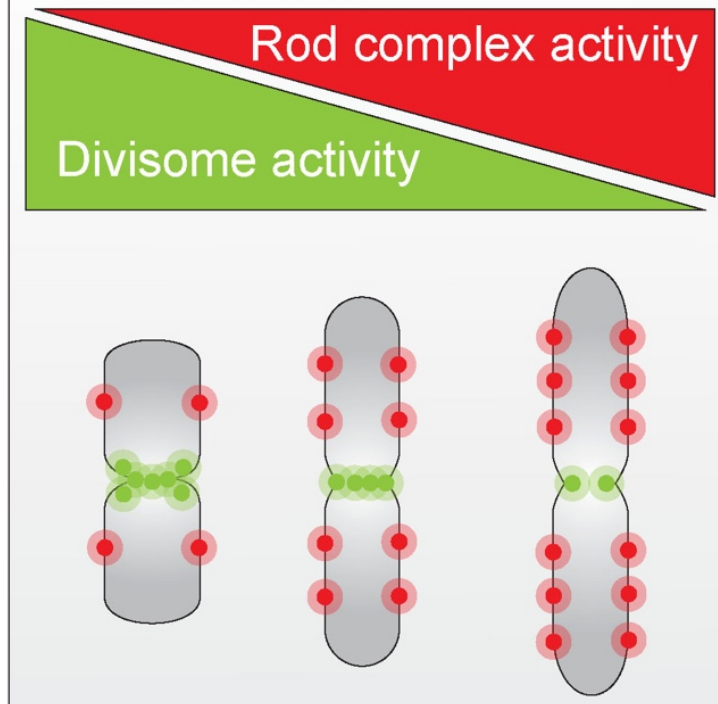

Cell shape

\begin{tabular}{|cll|}
\hline OM & Old PG & Divisome \\
$\mathrm{PS}$ & New PG & Rod complex \\
$\mathrm{IM}$ & &
\end{tabular}

Figure 6: Septal PG architecture and divisome activity modulate bacterial morphogenesis. (A) A wide range of different SPG architectures can result from the same division machinery through altering rates of cell wall synthesis and hydrolysis. Constrictive mode of cell division where OM and IM invaginate at similar velocities, a phenotype commonly associated with C. crescentus, is the result of lower SPG synthesis rates. Here, the septal cell wall displays a V-shaped wedge where no SPG plates are present. In contrast, inhibition of SPG hydrolysis causes a temporal separation of IM and OM constriction leading to septation. These septa are reminiscent to those of Gram-positive bacteria such as $S$. aureus, displaying two distinctive plates of sPG linked together by a PG bridge. Wild-type E. coli displays a mixed constrictionseptation phenotype resulting in a partial septum at later stages of the division process. (B) The activities of the two major synthetic cell wall machineries, the Rod complex and the divisome, are anticorrelated, likely due to competition for limited substrate (lipid II). Balance of their relative activities determines the shape of the cell division site and the resulting poles they form. Cells with higher Rod complex activity are thinner and form pointier poles, while cells with elevated divisome activity are shorter and wider with blunt poles. 